\title{
MASTER
}

WFPS-TME-089

MAY 1978

\section{FEEDBACK CONTROL OF VERTICAL INSTABILITY IN TNS}

\author{
ERIC R, FRANTZ \\ CARNEGIE-MELLON UNIVERSITY
}

COOPERATIVE GRADUATE EDUCATION PROGRAM IN FUSION TECHNOLOGY ADMINISTERED FOR THE U.S. DEPARTMENT OF ENERGY

BY THE WESTINGHOUSE ELECTRIC CORPORATION

CONTRACT EG-77-C-02-4231-A000 


\section{DISCLAIMER}

This report was prepared as an account of work sponsored by an agency of the United States Government. Neither the United States Government nor any agency Thereof, nor any of their employees, makes any warranty, express or implied, or assumes any legal liability or responsibility for the accuracy, completeness, or usefulness of any information, apparatus, product, or process disclosed, or represents that its use would not infringe privately owned rights. Reference herein to any specific commercial product, process, or service by trade name, trademark, manufacturer, or otherwise does not necessarily constitute or imply its endorsement, recommendation, or favoring by the United States Government or any agency thereof. The views and opinions of authors expressed herein do not necessarily state or reflect those of the United States Government or any agency thereof. 


\section{DISCLAIMER}

Portions of this document may be illegible in electronic image products. Images are produced from the best available original document. 


\section{ACKNOWLEDGEMENT}

This work was performed for the U.S. Department of Energy, under Contract EG-77-C-02-4231-A000, Cooperative Graduate Education Program in Fusion Technology. Reproduction, translation, publication, use and disposal, in whole or in part, by or for the United States Government is permitted.

The author is indebted to many people for the contributions that made this work possible. Special thanks go to my advisors: Dr. Tien-Fang Yang, for the topic proposal and his guidance in this project; and Dr. Yung-An Chao, for his continued help and advice. I would also like to thank my fellow graduate students, Mike Culbert, John Easoz, John McCowan, and Todd Pridgeon, and countless others at Westinghouse for making my stay enjoyable and enlightening. Finally, I would like to express my gratitude to $\mathrm{Dr}$. Daniel Klein of Westinghouse and $\mathrm{Dr}$. J. Fletcher Osterle of Carnegie-Mellon for making my participation in this graduate education program possible.

\section{LEGAL NOTICE}

This report was prepared as an account of Government sponsored work. Neither the United States, nor the Administration, nor any person acting on behalf of the Administration:

A. Makes any warranty or representation, expressed or implied, with respect to the accuracy, completeness, or usefulness of the information contained in this report, or that the use of any information, apparatus, method or process disclosed in this report may not infringe privately owned rights; or

B. Assumes any liabilities with respect to the use of, or for damages resulting from the use of any information, apparatus, method, or process disclosed in this report. 
WFPS-TME-039

MAY 1978

FEEDBACK CONTROL OF VERTICAL INSTABILITY IN TNS

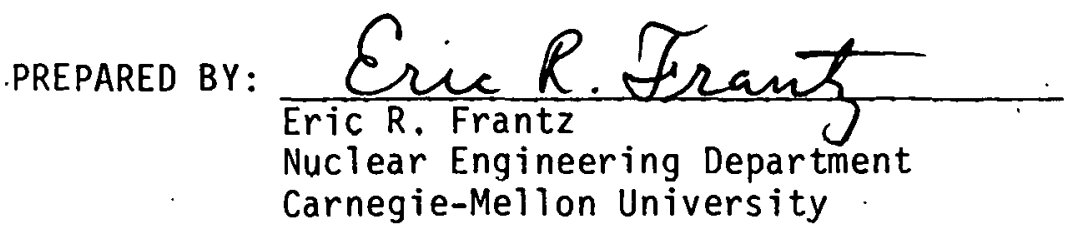

REVIEWED BY:
Westinghouse Electric Corp.

APPROVED BY:

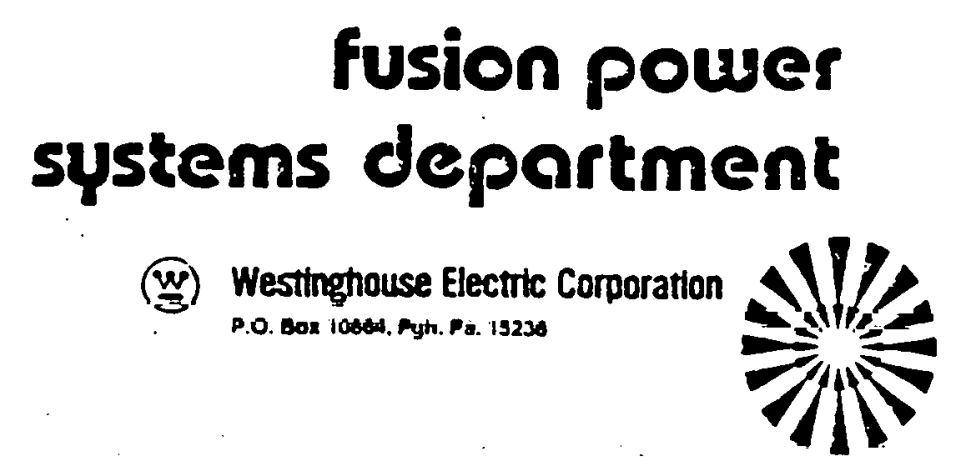




\section{ABSTRACT}

Due to the unfavorable curvature of the vertical vacuum magnetic field, elongated plasmas are vertically unstable when the elongation, $\varepsilon$, becomes too large. The TNS (The Next Step) tokamak, as evolved in the Westinghouse-ORNL studies has an inside- $D$ configuration $(\varepsilon=1.6, A=5 / 1.25=4$ ) characterized by an average decay index $n \approx-0.75$ at the plasma flux surface near the magnetic axis and is. vertically unstable with a growth rate $\gamma_{0} \sim 10^{5} \mathrm{sec}^{-1}$. Eddy currents produced in the vacuum vessel wall will slow this instability to growth rates $\gamma_{0} \sim 10^{2}$ $\mathrm{sec}^{-1}$ provided there are no transverse insulating gaps in the vessel wall.

A matrix equation has been developed for calculating the eddy currents induced in the EF coils and their stabilizing effect. Growth rates computed numerically have been fitted to the analytic shell model expression for $\gamma$ over a wide range of decay indexes $\left(-n \propto \gamma_{0}^{2}\right.$ varying from 0 to 1.6). Agreement between the numerical and analytic values is within $5 \%$ over the decay index range for three different series-parallel EF coil configurations. The coils are found to reduce the vertical growth rate for TNS to less than $1 \mathrm{sec}^{-1}$.

Control theory for feedback systems with and without delay time is presented and possible plasma position detectors are discussed. For a plasma current of 6.1 MA, the controller peak power requirements using separate controller circuits are $\sim 1 M W$ depending upon EF coil configurations and time delay. This feedback system is designed to stabilize a maximum plasma excursion of $10 \mathrm{~cm}$ from the midplane with delay times up to $2 \mathrm{sec}$. 
TABLE OF CONTENTS

Section No.

Page No.

1.0 INTRODUCTION

$1-1$

2.0 GROWTH RATE OF VERTICAL INSTABILITY WITH NO STABILIZING

$2-1$ MECHANISM

2.1 GROWTH RATE UTILIZING PLASMA EQUATION OF MOTION 2-1

2.2 APPLICATION TO TNS 2-3

3.0 STABILIZING EFFECT OF A PERFECT RESISTIVE WALL ON THE 3-1 VERTICAL INSTABILITY

3.1 VECTOR POTENTIAL OF THE PERTURBED PLASMA 3-1

3.2 EDDY CURRENTS AND THE INDUCED MAGNETIC FIELD 3-3 DUE TO THE FIRST WALL

3.3 APPLICATION TO TNS 3-9

4.0 STABILIZING EFFECT OF THE EQUILIBRIUM FIELD COILS ON * 4-1 THE VERTICAL INSTABILITY

4.1 DEVELOPMENT OF THE EQUATION OF MOTION AND THE EF $4-2$ COIL CIRCUIT EQUATIONS

4.2 APPLICATION TO TNS

$5.0^{\circ}$ FEEDBACK CONTROL OF THE VERTICAL INSTABILITY

5.1 CONTROL THEORY FOR FEEDBACK CONTROL OF THE 5-2 VERTICAL INSTABILITY

5.2 PLASMA POSITION DETECTORS 5-7

5.3 DESIGN FOR FEEDBACK CONTROL OF VERTICAL 5-10 INSTABILITY IN TNS

6.0 CONCLUSIONS $6-1$

7.0 REFERENCES $7-1$ 


\section{LIST OF FIGURES}

Figure No.

Page No.

$1-1$

Illustrations of Vertical Stability in Relation with

$1-2$ the Curvature of the Vertical Magnetic Field.

2.1-1 The Toroidal $(r, \phi, z)$ and Poloidal $(p, \theta)$ Coordinate

$2-2$ Systems With Vertically Elongated Ellipse $b>a$.

2.2-1 Vacuum Field for TNS Showing Position of Plasma and EF

$2-4$ Coils. Currents in EF Coils Indicated in Amp-Turns for $I_{p}=6.1 \mathrm{MA}, \beta=15 \%$.

3.2-1 Path of Integration for Eddy Current Circuit Equation. The Contours Form a Plane slicing the Shell at Constant

$3-6$ $x$.

3.3-1 Growth Rate With Stabilizing Effect of First Wall vs Decay Index.

4.1-1 The Parallel EF Coil Pair Connection Showing Equilibrium $4-3$ Current $I_{0}$ and Induced Current $I_{i}$ due to Vertical Motion of Plasma. The Plasma Current is Opposite in Direction to $I_{0}$ in al1 EF Coils Except the EF-D Coil. The Direction of $I_{j}$ is the same in all Coils for the Positive Perturbation छ̧z. In Practice, Each Top (Bottom) Coil will Consist of $N_{j}>>1$ Turns and may be Connected in Series With Other Top (Bottom) Coils and Collectively in Parallel with those Below (Above).

4.1-2 Graphical Analysis of Growth Rate for EF Coil Configura- 4-9 tions. Sketch of Eq. (4.1-25) for $N=5$.

4.2-1 Growth Rate of Vertical Instability vs Decay Index for 4-15 Different Parallel EF Coil Configurations when Other Plasma Parameters are Held Constant.

5.3-1 Typical Control Circuit for Feedback Control System • 5-13 Illustrating Series Connection for Controller. 


\section{LIST OF TABLES}

Table No.

Page No.

2.2-1 KEY PARAMETERS OF THE EQUILIBRIUM PLASMA USED TO

$2-6$

COMPUTE GROWTH RATE OF VERTICAL INSTABILITY IN TNS

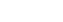

WITH NO STABILIZING INDUCED CURRENTS

4.2-1 INDUCTANCE MATRIX $M-\left(10^{2} H\right)$ FOR TNS REFERENCE

$4-12$

$4.2-2$

DATA FOR EF COIL PAIRS

4-13 


\subsection{INTRODUCTION}

It is known that plasmas elongated in the direction of the axis of symmetry may be unstable with respect to a vertical perturbation when the elongation exceeds a certain limit. This report serves to examine this apparent problem in general and to apply the resulting theory to the ORNL-Westinghouse TNS inside- $D$ reference plasma $\left(R_{p}=5 \mathrm{~m}, a=1.25 \mathrm{~m}, \varepsilon=b / a=1.6\right)^{(1)}$. The 0ak Ridge/Westinghouse contribution to TNS (The Next Step) Project is a study of various inside-D configuraliun tokanaks, most of which incorporate superconducting toroidal field coils in the design.

The benefits of the vertically elongated plasma shape have been identified and can be summarized as follows ${ }^{(2-6)}$ : (1) relatively higher $B$ than that of circular shape can be achieved; (2) elongation with triangular shape will help stabilization of the localized modes; and (3) the Kruskal-Shafranov limit for the internal kink mode on the plasma axis $q(0) \geq 1$ can be relaxed slightly. Experimentally, $\beta$ as high as $50 \%$ has been achieved for 20 MHD periods or $50 \mu$ s in the Belt Pinch $^{(4)}$.

Noncircular plasmas may become unstable in axisymmetric modes $(3,5)$. Higher order modes may he excited, but are ignored here. We are concerned with the most dangerous mode, the vertical rigid displacement of the plasma. This mode can be illustrated by Figure 1-1. Figure 1-la shows a vertically stable plasma. If the plasma is displaced the amount $\xi \hat{z}$ above the equatorial plane $z=0$, it encounters a restoring force per unit length $I_{p} \times B_{r}(\xi)$ tending to restore the plasma to its original position. Figure $1-1 b$ is not stable. The plasma will encounter $a B_{r}(\xi)$ in the opposite direction due to the opposite curvature of the field lines so that $I_{p} \times B_{r}(\xi)$ is directed away from the equatorial plane.

For the large aspect ratio approximation, the degree of the vertical stability can be characterized by the decay index of the field on axis defined as 


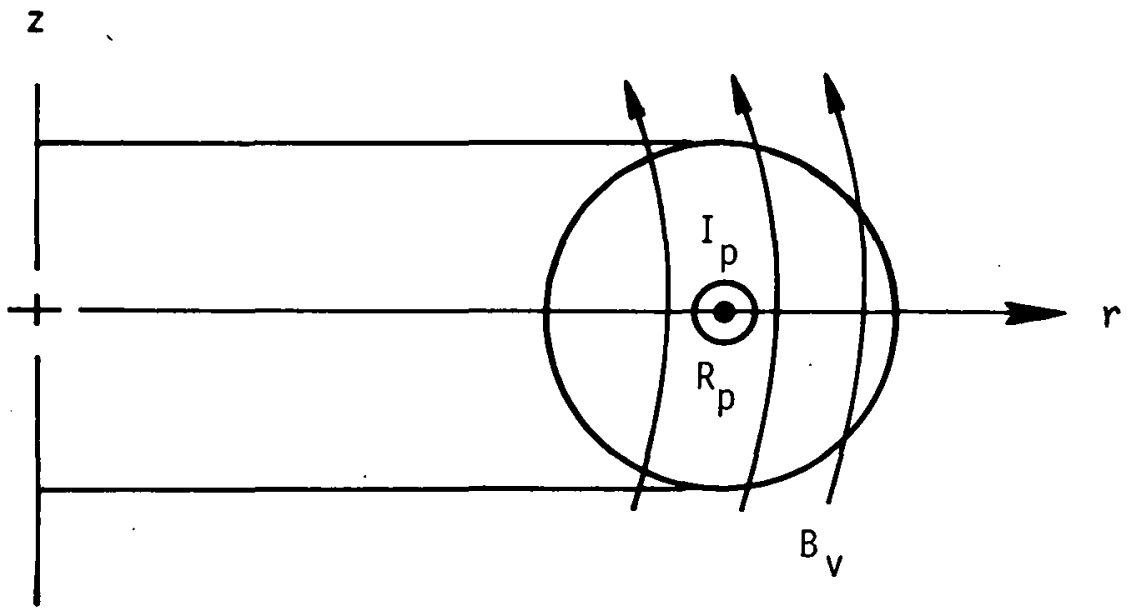

(a) Vertically Stable Circular Plasma (Right Magnetic Field Curvature)

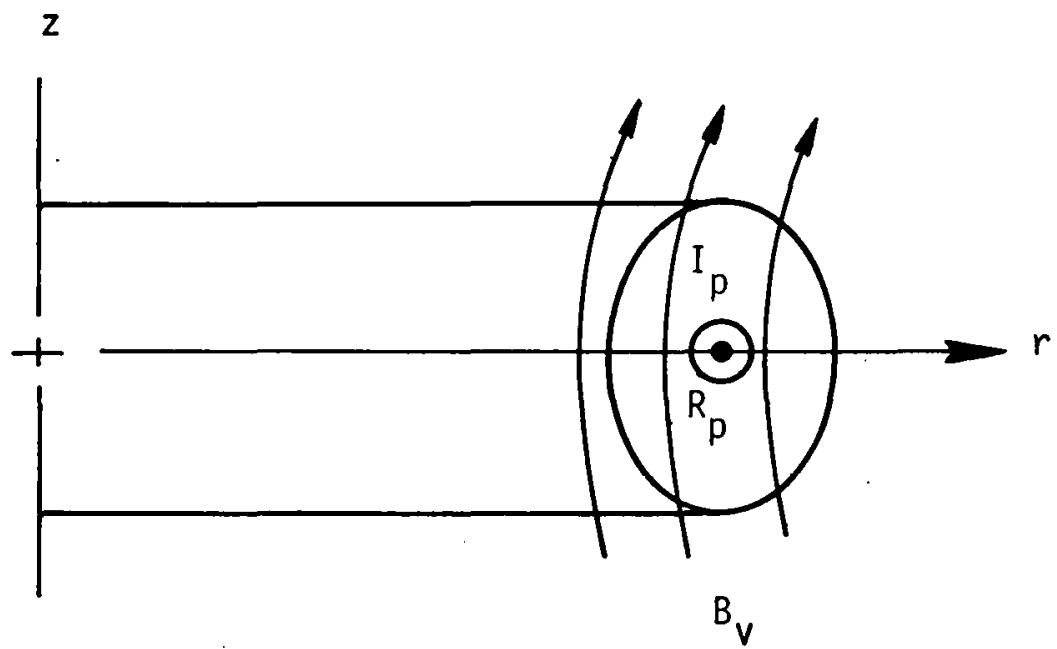

(b) Vertically Unstable Elongated Plasma (Wrong Magnetic Field Curvature)

Figure 1-1. Illustrations of Vertical Stability In Relation with the Curvature of the Vertical Magnetic Field. 


$$
n=-\frac{r}{B_{z}}:\left.\frac{\partial B_{z}}{\partial r}\right|_{r=R_{p}}
$$

The condition to insure vertical stability is $\eta>0$. The criterion for horizontal stability is $n<3 / 2(7)$. This instability has also been evaluated more generaliy by taking into account the plasma shape, diffused pressure, and current profiles for more realistic aspect ratios by using the energy principle and small perturbation theory. A triangular shape was found to be more stable and a critical elongation was determined $(3,6)$. However, a general method for predicting the growth rate of the vertical displacement when taking into account the effect of the equilibrium field maintaining coils has not yet been given. That is the purpose of this report. Since we are interested in the order of magnitude of the growth rate, and also for simplicity and for the convenience of feedback control analyses, we keep the approximate approach as has just been described.

The context is conveniently divided into five major sections. In Section 2, the growth rate for vertical motion is analyzed in the approximation that the plasma moves as a rigid body unaided by stabilizing eddy currents in external conductors. The averaged decay index near the magnetic axis, a measure of the vertical vacuum field curvature, ( $n \sim-0.8$ for TNS) is an important parameter in determining this growth rate. The rising time (inverse growth rate) for TNS at $I_{p}-6.1 \mathrm{MA}$ is $\sim 3 \mathrm{\mu sec}$ under these conditions.

Induced eddy currents fortunately slow this growth rate down to more controllable values. The effect of a perfect (i.e., without gaps) resistive wall on the growth rate is examined in Section 3 . The analysis is carried out to the first order in $a / R$ and results in a $\sin \theta$ (plus a small quadrupole) eddy current distribution in the wall (here $\theta$ defines the poloidal angle). This gives rise to a stabilizing induced radial field that modifies the previous equation of motion and increases the rising time in TNS to $\sim 30 \mathrm{~ms}$ for a $12 \mathrm{~mm}$ thick stainless steel vacuum vessel, continuous in the toroidal direction $(\phi)$. However, the vessel wall may require resistive gaps to allow the external toroldal and poloidal fields to diffuse more rapidly inward to the plasma making the above assumption less realistic. 
The stabilizing effect of the discrete equilibrium field coils (EF coils) is presented in Section 4. Here the EF coils above the equatorial plane are connected in parallel to those below the plane. In this manner, stabilizing eddy currents are free to flow in the coils and do not cancel one another. Three different series-parallel configurations are examined that satisfy the up-down parallel requirements. The resulting circuit equations and the plasma equation of motion simplify to an eigenvalue equation for the induced EF coil currents. This eigenvalue equation is solved numerically, yielding the EF coil current amplitudes and the growth rate of the vertical instability. The EF coils are found to have a strong stabilizing influence that lengthens the rising time in TNS to several seconds, the exact value depending upon which of the three configurations is used. It is also found that the eddy current distribution in the EF coils resembles the $\sin \theta$ distribution found in the resistive wall. The analogy is so strong that a numerical fit of the growth rate to the analytic shell growth rate formula can result in less than a $5 \%$ deviation over a wide range of decay indexes (0 thru -1.6).

Section 5 deals with the active feedback control of the vertical instability. The analysis is simplified by exploiting the already demonstrated shell-like effect of the EF coils. We consider both instantaneous and delayed feedback control and specify controller gain requirements, maximum delay time, and controller power requirements. Possible plasma position detectors are examined and a conceptual feedback design for TNS is presented. Power requirements for the feedback system are modestly low $(\sim 1 \mathrm{MW})$ for a $10 \mathrm{~cm}$ maximum plasma excursion from the midplane. Three control loops separate from the EF coils are envisioned in this design, and will control the plasma under the above conditions for a plasma current of $6.1 \mathrm{MA}$, the $15 \%$ \& limit imposed on TNS. 


\subsection{GROWTH RATE OF VERTICAL INSTABILITY WITH NO STABILIZING MECHANISM}

\subsection{GROWTH RATE UTILIZING PLASMA EQUATION OF MOTION}

Let us consider a plasma carrying a current $I_{p}$ and situated in an external vacuum field $B_{v}$ whose curvature is characterized by a decay index $n$. In equilibrium at $z=0$, all electromagnetic and pressure gradient forces balance and there is no tendency for all or any part of the plasma toward motion.

Now let this plasma be displaced the amount $\xi \hat{z}$ subject to the constraint that the currents in the plasma and external conductors remain fixed. Only $\mathrm{J}_{\phi} \times \mathrm{B}_{V}$ forces act on the plasma since the poloidal fluxes generated by the plasma current moves with the displaced plasma and gives no net contribution. Expanding $B_{v}$ in a Taylor series about $z=0$ and keeping the first order

$$
\begin{aligned}
& B_{z}(\xi)=B_{z}(0)+\left.\frac{\partial B_{z}}{\partial z}\right|_{0} \xi \\
& B_{r}(\xi)=B_{r}(0)+\left.\frac{\partial B_{r}}{\partial z}\right|_{0} \xi
\end{aligned}
$$

There is no net radial force ${\underline{J_{\phi}}}_{\partial B} \times \delta_{Z} B_{Z}(\xi)$ for first order since $\underline{\nabla} \cdot \underline{B_{v}}=0$ requires $\left.\frac{\partial B}{\partial z}\right|_{0}=0$.

Let us arbitrarily pick $J_{\phi}$ in the $-\hat{e}_{\phi}$ direction (see Figure 2.1-1) so $\mathrm{B}_{v}$ must be upward to provide radial equilibrium. Dropping the subscripts and $\frac{v}{4}$ ing $B_{r}(0)=0$, we may then express the vertical force on the plasma by

$$
F_{z}=\int J_{\phi}(r, z) \frac{\partial B}{\partial z} \xi d V
$$

Using $\underline{\nabla} \times \underline{B_{v}}=0$, Equation $(2.1-3)$ may be rewritten as

$$
F_{z}=-\int J_{\phi}(r, z) \frac{B_{z}{ }^{n}}{r} \xi d V
$$




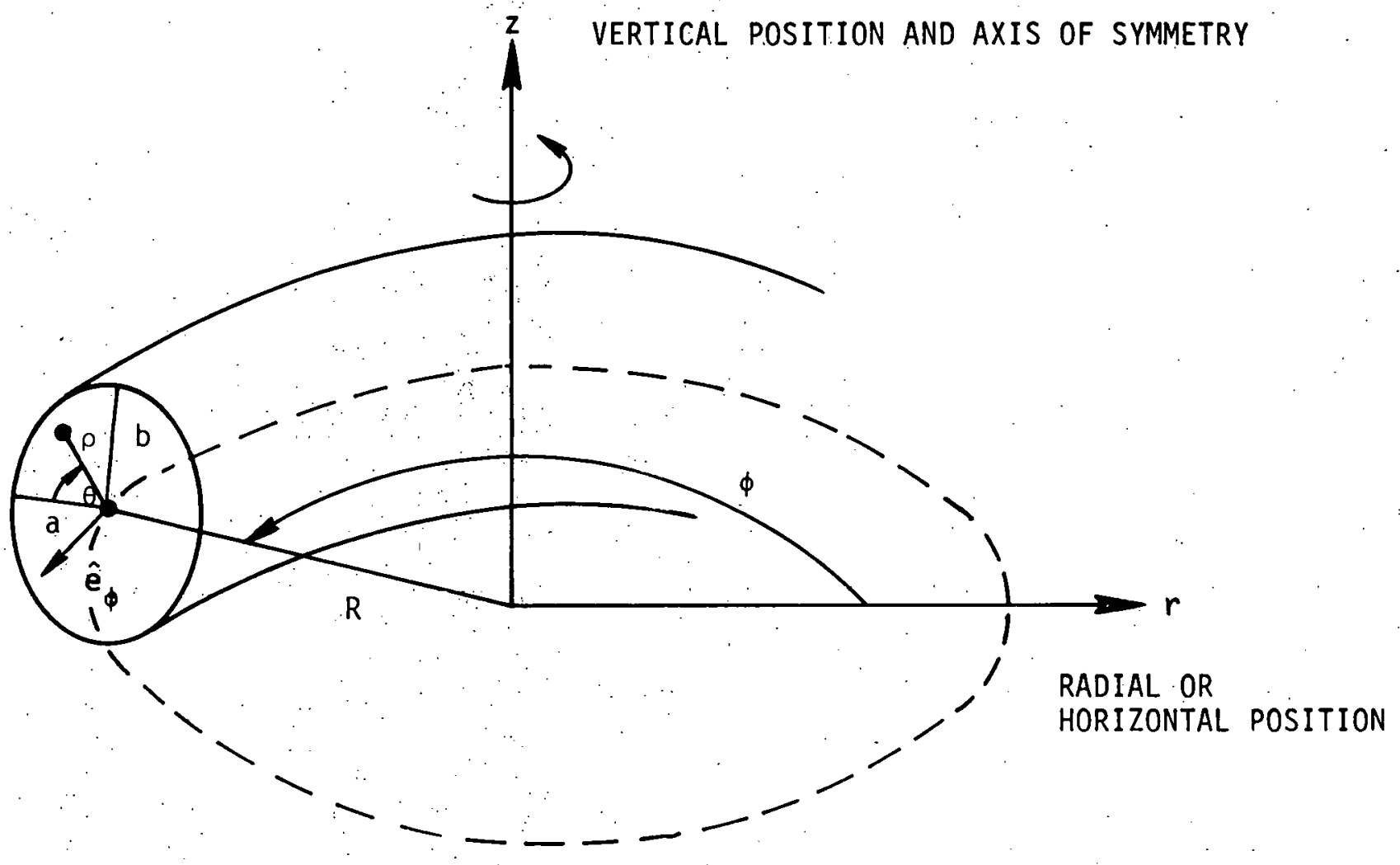

Figure 2.1-1. The Toroidal $(r, \phi, z)$ and Poloidal $(\rho, \theta)$ Coordinate Systems With Vertically Elongated Ellipse $b>a$. 
For the large aspect ratio approximation, Eq. (2.1-4) reduces to the equation of motion of the plasma

$$
m \frac{d^{2} \xi}{d t^{2}}=-\frac{{ }^{B} z}{R_{p}} I_{p} \xi
$$

where $m$ is the mass per unit length of the plasma column and the decay index, $\eta$, is defined as

$$
\eta=-\left.\frac{r}{B_{z}} \frac{\partial B_{z}}{\partial r}\right|_{r=R_{p}}
$$

For a time dependence $\xi=\xi_{0} e^{\gamma t}$. Eq. (2.1-5) leads to the dispersion relation

where

$$
r^{2}-r_{0}^{2}=0
$$

$$
r_{0}^{2} \equiv-\frac{n B_{z} I_{p}}{m R_{p}}
$$

For $n<0$, the dominant mode grows exponentially at a rate $\gamma=+\gamma_{0}$ and for $\eta>0$, the plasma is stable.

\subsection{APPLICATION TO TNS}

A plot of the magnetic flux lines of the external vacuum field is presented in Figure 2.2-1. The equilibrium field coils (EF coils) giving rise to this field are indicated by the squares numbered in order of increasing radius.

The EF coil currents required for $I_{p}=-6.1 \mathrm{MA}$ and $B=15 \%$ are $I_{E F-I}=0.26 \mathrm{MA}$ (coils I through 4), $\mathrm{I}_{E F-D}=-0.82 \mathrm{MA}$ (coil 5), and $\mathrm{I}_{E F-0}=0.57 \mathrm{MA}$ (coils 6 through 10). Induced and control currents discussed in Sections 4 and 5 are indicated above the coils. All currents are given in total amp-turns and flow in the positive sense $\left(+\hat{e}_{\phi}\right)$ with the exception of the divertor coil (5) and plasma currents which flow in the opposite direction. The vacuum field $B_{v}$ is therefore directed upward by our convention. The plasma boundary is also indicated in the figure and occupies an area of approximately $8 \mathrm{~m}^{2}$. A majority of the current, however, occupies an area of $\sim 2.5 \mathrm{~m}^{2}$ located in the region to 


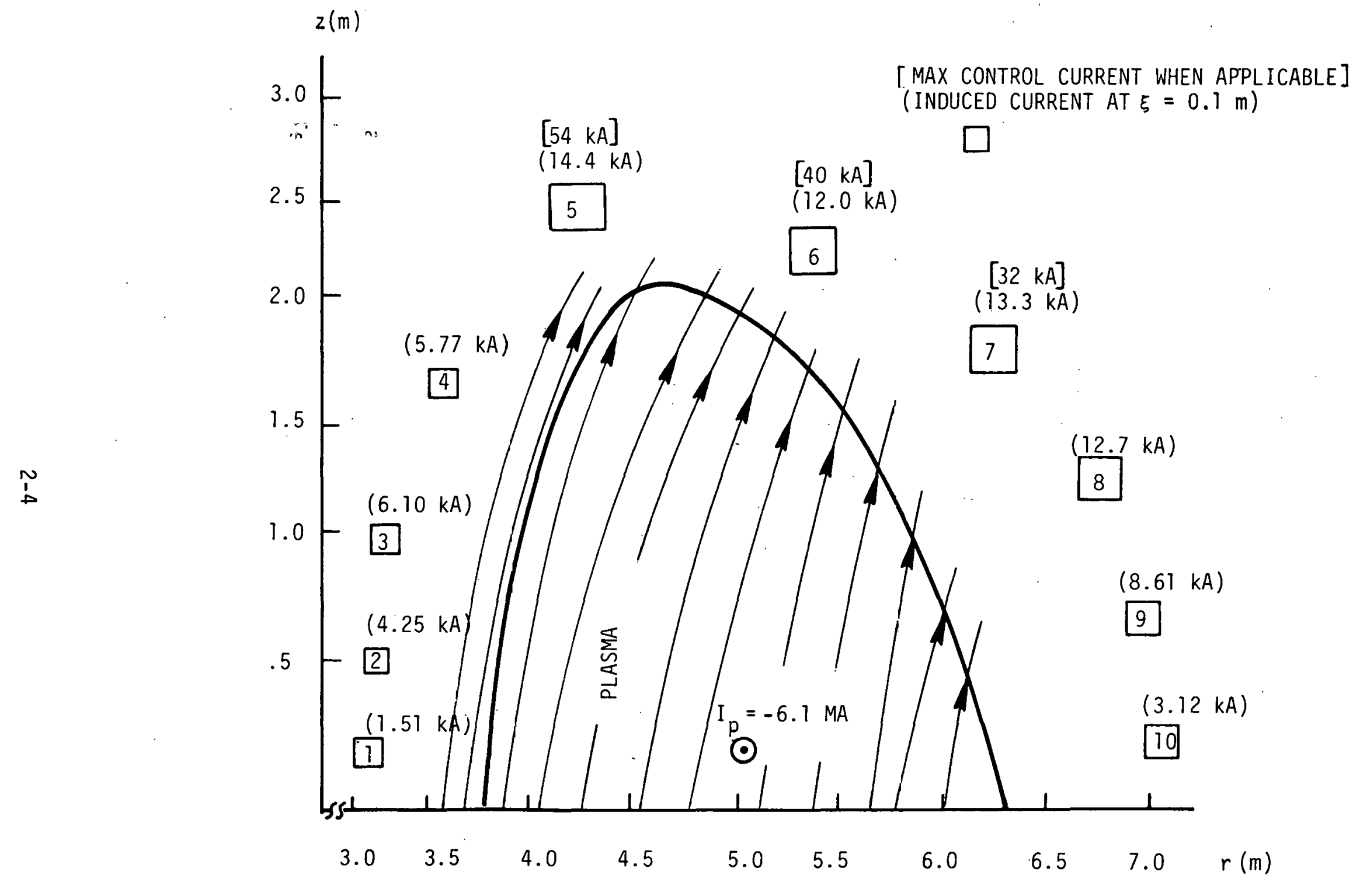

Figure 2.2-1. Vacuum Field for TNS Showing Position of Plasma and EF Coils. Currents in EF Coils Indicated in Amp-Turns for $I_{p}=-6.1 \mathrm{MA}$, $\beta=15 \%$. 
the outside of major radius $R_{p}=5.0 \mathrm{~m}$. The magnetic axis is displaced slightly beyond this point $R_{m} \sim 5.3 \mathrm{~m}$. The computations are in accordance with Peng's poloidal field system design specifications for TNS $(1)$.

The key plasma parameters and equilibrium vacuum field data used in the computation of the vertical growth rate without stabilizing effects are presented in Tabie 2.2-1. The growth rate computed by Eq. (2.1-7) (from the equation of motion) is $\gamma=\gamma_{0}=3.58 \times 10^{5} \mathrm{~s}^{-1}$, corresponding to a rising time of $2.8 \mu \mathrm{s}$. For comparison, the growth rate is also estimated from an energy principle. For a large aspect ratio elliptical plasma with a quasi-constant current profile and conducting wall located at infinity, Haas has found that the change of potential energy due to vertical displacement is given by ${ }^{(8)}$

$$
\delta W=-\frac{u_{0} \xi^{2} I^{2} R_{o} a b}{\left(b^{2}+a^{2}\right)^{1 / 2}}\left\{1+\frac{1}{2}\left(\frac{\beta}{\beta_{c}}\right)^{2}\right\}
$$

where the limiting $\beta$ is defined as

$$
\beta_{c}=\frac{1}{A q_{0}^{2}} \frac{3+\left(\frac{a}{b}\right)^{2}}{\left(1+\left(\frac{a}{b}\right)^{2}\right)^{2}} \text {. }
$$

The growth rate can be estimated from

$$
r=\left(-\frac{2}{M_{p}} \frac{\delta W}{\xi^{2}}\right)^{1 / 2}
$$

which yields $3.40 \times 10^{5} \mathrm{~s}^{-1}$ for TNS. The results for both methods are in agreement.

A configuration that is unstable in a matter of a few us would be near impossible as well as impractical to control. Response and reprocessing times less than 1 us would be required, but such technology is still in developmental stages. Power supply requirements of a controller may be astronomical since amplifier rise times $\sim \mu s$ would be required. 
TABLE 2.2-1

KEY PARAMETERS OF THE EQUILIBRIUM PLASMA USED TO COMPUTE GROWTH RATE OF VERTICAL INSTABILITY IN TNS WITH NO STABILIZING INDUCED CURRENTS

$$
\begin{aligned}
& R_{p}=R_{0}=5 \mathrm{~m} \\
& \mathrm{a}=1.25 \mathrm{~m} \\
& b=2.0 \mathrm{~m} \\
& n=-0.76\left(a t R_{p}\right) \\
& B_{z}=0.57 \mathrm{~T}\left(\text { at } R_{p}\right) \\
& I_{p}=6.1 \mathrm{MA} \\
& \beta^{p}=0.15 \\
& \bar{n}=1.25 \times 10^{20} \mathrm{~m}^{-3} \text { (average density) } \\
& a_{p}=7.85 \mathrm{~m}^{2} \\
& \bar{\mu}=4.2 \times 10^{-27} \mathrm{~kg} \\
& \text { (effective particle mass for } \\
& m=\bar{n} \bar{\mu} a_{p}=4.12 \times 10^{-6} \mathrm{~kg} / \mathrm{m} \\
& M_{p}=2 \pi R_{p}{ }^{p}=0.130 \times 10^{-3} \mathrm{~kg}
\end{aligned}
$$

VARIATION OF $B_{2}$ AND $-n$ ALONG MAJOR RADIUS

\begin{tabular}{|l|l|l|l|}
$r(m)$ & $-n$ & $B_{z}(T)$ & $\frac{-n B_{Z}}{r}(T / m)$ \\
\hline 4.5 & 1.03 & 0.517 & 0.118 \\
5.0 & 0.763 & 0.568 & 0.0867 \\
5.5 & 0.478 & 0.603 & 0.0524
\end{tabular}


Fortunately, it has been demonstrated that the rise time can be reduced significantly by the eddy current induced in the first wall which will be considered in the next section. 


\subsection{STABILIZING EFFECT OF A PERFECT RESISTIVE WALL ON THE VERTICAL INSTABILITY}

The resistive shell has been treated in cylindrical coordinates and the rise time has been found to be reduced by three orders of magnitude. In the treatment given in the following subsection, the toroidal effect is taken into account.

\subsection{VECTOR POTENTIAL OF THE PERTURBED PLASMA}

The vector potential of a current loop of radius $R_{p}$ carrying a current $I_{p}$ is well known $(9,10)$

$$
A_{\phi}=\frac{\mu_{0} I p}{\pi}\left(\frac{R_{p}}{r k^{2}}\right)^{1 / 2}\left[\left(1-\frac{1}{2} k^{2}\right) k\left(k^{2}\right)-E\left(k^{2}\right)\right]
$$

where $K$ and $E$ are the complete elliptic integrals of the first and second kind, respectively, with argument

$$
k^{2}=\frac{4 R_{p} r}{\left(R_{p}+r\right)^{2}+z^{2}}
$$

Transforming to the poloidal (polar) coordinates

$$
\begin{aligned}
& r=R_{p}+\rho \cos \theta \\
& z=\rho \sin \theta
\end{aligned}
$$

and expanding the elliptic integrals about $R_{p}$, one can derive

$$
\dot{A}_{\phi}=\frac{\mu_{0} I_{p} R_{p}}{2 \pi r}\left[\ln \left(\frac{8 R_{p}}{\rho}\right)-2\right]+\frac{\mu_{0} I_{p}}{4 \pi r}\left[\ln \left(\frac{8 R_{p}}{\rho}\right)-2\right] \rho \cos \theta
$$

to first order in $\rho / r$. In the limit $R_{p} \rightarrow \infty$, with $R_{p} / r \rightarrow 1$, the spatial dependence of $A_{\phi}$ reduces tn

$$
A_{\phi}=-\frac{\mu_{0} I p}{2 \pi} \ln \rho
$$


the well known result for the long straight wire. Thus, the $\rho \cos \theta$ term in Eq. (3.1-3) expresses the toroidal effect to first order in $\rho / R_{p}$.

It can be shown that the vector potential of the plasma is related to the poloidal flux function of the plasma, $\Psi_{p}$, by

$$
A_{\phi}=\frac{\psi p}{2 \pi r} \text {. }
$$

Using a form for $\Psi_{p}$ given by Mukhovatov and Shafranov ${ }^{(7)}$ for the large aspect ratio circular plasma, the vector potential for finite pressure can be written as

$$
\begin{aligned}
A_{\phi}= & \frac{\mu_{0} I_{p} R_{p}}{2 \pi r}\left[\ln \left(\frac{8 R_{p}}{\rho}\right)-2\right]+ \\
& \frac{\mu_{0} I p}{4 \pi r}\left[\ln \left(\frac{8 R_{p}}{\rho}\right)-1+\frac{a^{2}}{\rho^{2}}\left(\Lambda+\frac{1}{2}\right)\right] \rho \cos \theta
\end{aligned}
$$

to first order in $\rho / r$ for $\rho>a$. Here, $\Lambda$ is the asymmetry factor defined by

$$
\Lambda=\beta_{\theta}+\frac{1}{2}-1 \text {. }
$$

where $\beta_{\theta}$ is the poloidal $\beta$ and $l_{i}$ is the inductance per unit length of the plasma. If the plasma is vertically perturbed the amount $\xi \hat{z}$, the cosine and sine laws permit us to reference the vector potential in terms of the unperturbed position by the substitutions

$$
\begin{gathered}
\rho \rightarrow \rho\left(1-\frac{\xi}{\rho} \sin \theta\right) \\
\cos \theta \rightarrow \cos \theta\left(1+\frac{\xi}{\rho} \sin \theta\right)
\end{gathered}
$$

given to first order in $\xi / \rho$. This linearized form shows that $\rho \cos \theta$ remains unchanged to first order.

Using Eq. (3.1-8) in Eq. $(3 \cdot 1-6)$ and expanding

$$
\frac{1}{r}=\frac{1}{R_{p}}\left(1-\frac{\rho \cos \theta}{R_{p}}\right)
$$


gives the change in the vector potential for the plasma

$$
\delta \dot{A}_{\phi}=\frac{\mu_{0} I p}{2 \pi}[1-f(\rho) \cos \theta] \frac{\xi}{\rho} \sin \theta
$$

where

$$
f(\rho)=\frac{\rho}{R_{p}}\left[\frac{1}{2}-\frac{a^{2}}{\rho^{2}}\left(\Lambda+\frac{1}{2}\right)\right]
$$

expresses the first order correction due to toroidal effects and finite plasma size and pressure.

\subsection{EDDY CURRENTS AND THE INDUCED MAGNETIC FIELD DUE TO THE FIRST WALL}

Consider a first wall with conductivity $\sigma$ located at $\rho=\rho_{W}$ with thickness $\delta \ll \rho_{w}$. The perturbed vector potential Eq. (3.1-10) gives rise to an electric field in the wall $(9)$

$$
E_{\phi}=\frac{-\partial\left(\delta A_{\phi}\right)}{\partial t}
$$

that determines an eddy current density distribution

$$
\underline{j}_{w}=j_{0}\left[1-f\left(\rho_{w}\right) \cos \theta\right] \sin \theta \hat{e}_{\phi} .
$$

It has been assumed that this distribution is uniform over the wall thickness $\delta$. This approximation is valid provided the current diffusion time (or skin time)

$$
t_{s}=\frac{\mu_{0} \delta^{2} \sigma}{2}
$$

is small in comparison to the characteristic time associated with the perturbation (i.e., the rising time or the period of other modes). The magnetic permeability $\mu$ has been taken to be that of vacuum $\mu_{0}$. In the regions exterior to the wall ( $\rho>\rho_{W}$ or $\left.\rho<\rho_{W}\right)$ the induced field satisfies $\underline{\nabla} \times \underline{b}=0$. Thus, $\underline{b}$ is derivable from a scalar potential $\underline{b}=-\underline{\nabla} V$. Since $\underline{\nabla} \cdot \underline{b}=0, V$ satisfies Laplace's Equation $\nabla^{2} v=0$. The complete solution for $V$ can then be expressed in terms of the cylindrical harmonics 


$$
\begin{aligned}
V= & a_{0}+b_{0} \ln \rho+\sum_{n=1}^{\infty}\left(a_{n} \rho^{n}+\frac{c_{n}}{\rho^{n+T}}\right) \sin n \theta+ \\
& \left(b_{n} \rho^{n}+\frac{d_{n}}{\rho^{n+T}}\right) \cos n \theta
\end{aligned}
$$

where the coefficients remain to be determined by boundary conditions. Due to the uniformity of $j_{w}$ over its thickness, we may treat the shell as a conductor with a surface current per unit length

$$
\underline{k}=\underline{j}_{W} \frac{\delta}{2}
$$

flowing on the inside and outside of the shell and $\underline{b}=0$ within the wall internal. The boundary condition then becomes

$$
\hat{n} \times[\underline{b}]]=\mu_{0} \underline{k}\left[1-f\left(\rho_{w}\right) \cos \theta\right]
$$

where the double brackets $[[]]$ denote the difference in $\underline{b}$ at the boundary, and $\hat{n}$ is the normal to the wall surface. The toroidal effect appears again in Eq. (3.2-6) so that we may define $e_{\theta} \times e_{\rho}=e_{\phi}$. Using Eq. (3.1-4) thru Eq. (3.1-6) with $\underline{b}=-\nabla V$ allows us to match the coefficients of the cylindrical harmonic expansion and determine $\underline{b}$ uniquely. The solution is a series of multipole fields. Retaining the lowest two orders, the solution in the region inside the vessel, for $\rho<\rho_{w}$, can be written as

$$
\underline{b}=-\frac{\mu_{0} j_{0} \delta}{2} \hat{e}_{r}+\frac{\mu_{0} j_{0} \delta}{2 \rho_{w}} f\left(\rho_{w}\right)\left[\left(r-R_{p}\right) \hat{e}_{r}-z \hat{z}\right] \text {. }
$$

The second term is a quadrupole field whose contribution is to the order of $\rho_{W} / R_{p}$

The fields in the region outside the vessel, for $\rho>\rho_{w}+\delta$, are

$$
\underline{b}=-\frac{\mu_{0} j_{0} \delta}{2}\left\{\frac{\rho_{w}^{2}}{\rho^{4}}\left[\left(x^{2}-z^{2}\right) \hat{r}+2 x z \hat{z}\right]+\frac{\rho_{w}^{2}}{\rho^{3}} f\left(\rho_{w}\right)(x \hat{r}-z \hat{z})\right\}
$$

where $x=r-R_{p}$. This equation consists of dipole and quadrupole fields. 
The radial components of $\underline{b}$ inside the vacuum vessel is of opposite sign to $B_{r}$ and is stabilizing. The vertical force given in.Eq. (2.1-4) can now be modified as

$$
F_{z}=\int J_{\phi}(r, z)\left[-\frac{n B z}{r} \xi-\frac{\dot{j}_{0} j_{0} \delta}{2}\left(1-\frac{\rho}{\rho_{W}} f\left(\rho_{W}\right) \cos \theta\right)\right] d V
$$

Since the toroidal effect gives a contribution of order $a / R_{p}$ and the shift in the magnetic axis is also of this order, its net contribution to the integral is negligible. Thus, Eq. (3.2-9) can be re-expressed as

$$
\frac{d^{2} \xi}{d t^{2}}-\gamma_{0}^{2} \xi=-\frac{\mu_{0} j_{0} \delta}{2 m} I_{p}
$$

To determine the value of $j_{0}$, we will need to develop a circuit equation for the eddy current distribution. The equation will consist of three parts - (1) an emf induced by the shel1 (the LI contribution of the eddy currents), (2) an emf induced by the plasma (the $I_{p} \dot{M}$ contribution due to the plasma current motion), and (3) the resistance drop in the shell (the IR drop). The plasma current. $I_{p}$ is assumed constant so no $L_{p} \dot{I}_{p}$ term is present.

Consider the path depicted in Figure 3.2-1. The emf induced by the shell is

$$
e m f_{1}=\frac{-d \Phi_{s h}}{d t}
$$

where $\Phi_{\text {sh }}$ is the net flux enclosed by the path and is given by

$$
\Phi_{s h}=\frac{\mu_{0} j_{0} \delta}{2}\left[1-f\left(\rho_{w}\right) \cos \theta\right]\left(2 \rho_{w} l \sin \theta\right)
$$

The emf induced by the plasma motion is:

$$
e m f_{2}=-\frac{\partial}{\partial t} \oint_{\delta A_{\phi}} \cdot \underline{d l}
$$

or using Eq. $(3.1-10)$

$$
e m f_{2}=\frac{\mu_{0} I_{p}}{2 \pi \rho_{w}}\left(1-f\left(\rho_{w}\right) \cos \theta\right)\left(2 \rho_{w} l \sin \theta\right) \frac{d \xi}{d t}
$$




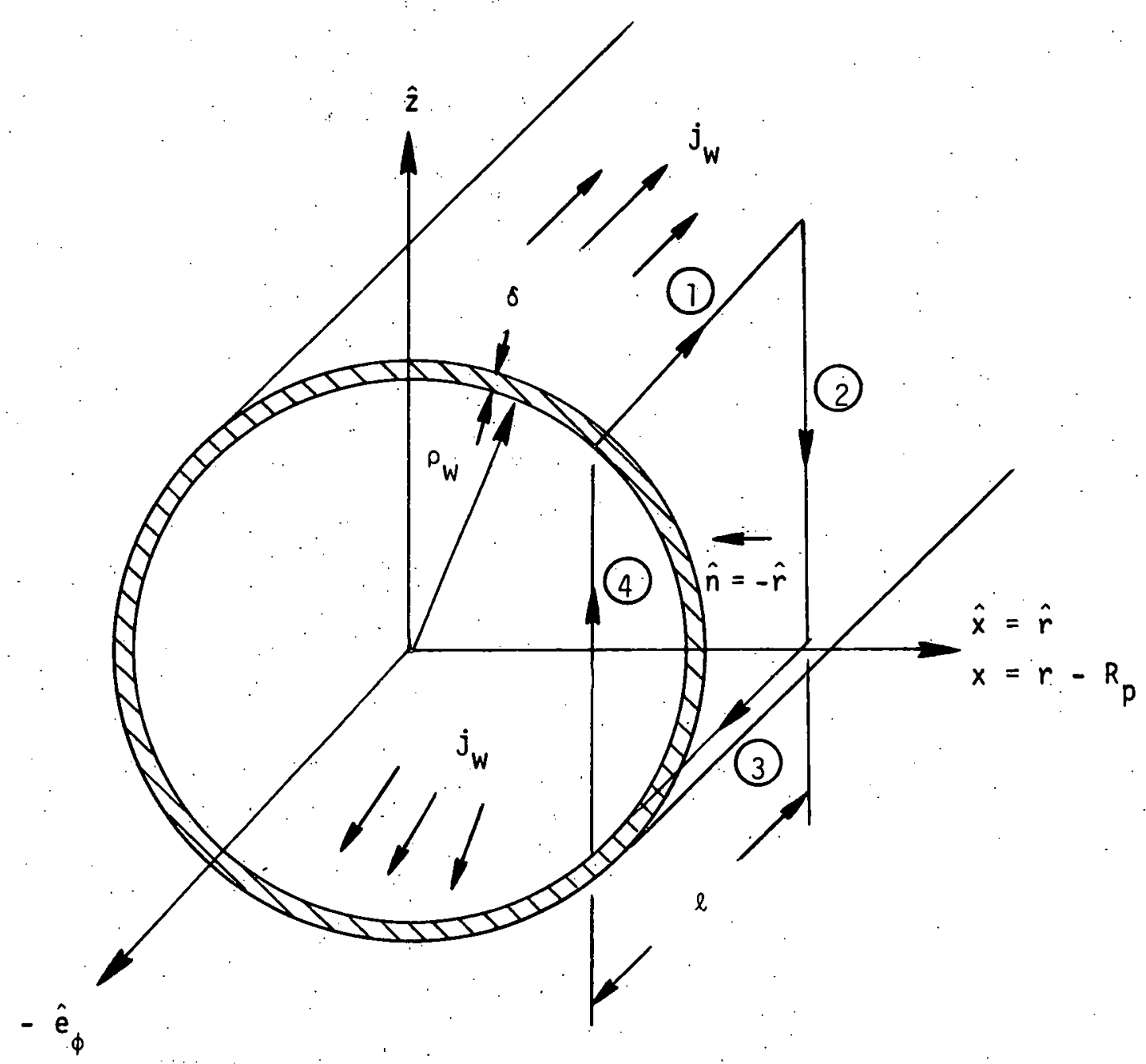

Figure 3.2-1. Path of Integration for Eddy Current Circuit Equation. The Contours Form a Plane Slicing the Shell at Constant $x$. 
with the partial becoming the total time derivative. The resistance drop is simply

$$
\mathrm{emf}_{2}=-\frac{1}{\sigma} \oint_{\underline{j_{w}}} \cdot \underline{\mathrm{dl}}
$$

or

$$
e m f_{3}=\frac{-j_{0}}{\sigma}\left(1-f\left(\rho_{w}\right) \cos \theta\right)\left(2 \rho_{w} l \sin \theta\right)
$$

Factoring out the common factor $\left(1-f\left(\rho_{w}\right) \cos \theta\right)\left(2 \rho_{w} \ell \sin \theta\right)$ and summing the emf's to zero gives

$$
\frac{j_{0}}{\sigma}+\frac{\mu_{0} \delta \rho_{w}}{2} \frac{d j_{0}}{d t}-\frac{\mu_{0} I_{p}}{2 \pi \rho_{w}} \frac{d \xi}{d t}=0
$$

independent of $\theta$.

Assuming a time dependence for $\xi$ and $j_{0}$ of the form $e^{z t}$, Eqs. $(3.2-10)$ and $(3.2-17)$ can be combined to eliminate $j_{0}$ and $\xi$ giving

$$
z^{2}-\gamma_{0}^{2}=\frac{\omega_{0}^{2} \tau z}{(1+\tau z)}
$$

where

$$
\omega_{0}^{2}=\frac{\mu_{0} I_{p}^{2}}{2 \pi m \rho_{w}^{2}}
$$

and

$$
\tau=\frac{\mu_{0} \delta \sigma \rho_{W}}{2}
$$

The first two terms in Eq. (3.2-17) are the IR drop and the LI of the shell. Thus, $\tau$ is the L/R time of the shell. For the perfectly conducting shell, $\tau \rightarrow \infty$ and $E q .(3.2-18)$ reduces to

$$
z^{2}=\omega_{0}^{2}-\gamma_{0}^{2}
$$


The dispersion relation, Eq. $(3,2-18)$, is a cubic equation and can be rewritten as

$$
z^{3}-\frac{z^{2}}{\tau}+\left(\omega_{0}^{2}-\gamma_{0}^{2}\right) z-\frac{\gamma_{0}^{2}}{\tau}=0
$$

We can investigate its stability properties by writting the Roth array

$$
\begin{array}{l|lll}
z^{3} & 1 & \omega_{0}{ }^{2}-\gamma_{0}{ }^{2} & 0 \\
z^{2} & 1 / \tau & -\gamma_{0}{ }^{2} / \tau & 0 \\
z & \omega^{2} & 0 & 0 \\
1 & { }^{2} & 0 & \\
\gamma_{0}^{2} / \tau & 0 & 0
\end{array}
$$

The number of sign changes in the first column of the Roth array dictates the number of unstable roots. Since we are concerned with $\gamma_{0}{ }^{2}>0(i . e ., n<0)$ there will be one unstable root $R_{e} z>0$ and two damped roots.

Typically, $\beta \sim 10^{-2} \mathrm{~s}$ and $\omega_{0}^{2} \sim \gamma_{0}^{2} \sim 10^{10} \mathrm{~s}^{-2}$ for MA currents. The solutions to the cubic Eq. $(3.2-22)$ or Eq. $(3.2-18)$ can then be well approximated (typically. with less than $1 \%$ error) for $\frac{1}{\tau^{2}} \ll\left|\omega_{0}{ }^{2}-\gamma_{0}{ }^{2}\right|$ by

$$
\begin{gathered}
z_{1}=\frac{\gamma_{0}{ }^{2}}{\tau\left(\omega_{0}{ }^{2}-\gamma_{0}{ }^{2}\right)} \\
z_{2,3}= \pm i\left(\omega_{0}^{2}-\gamma_{0}{ }^{2}\right)^{\frac{1}{2}}-\frac{1}{2}\left(z_{1}+\frac{1}{\tau}\right)
\end{gathered}
$$

The solution $z_{1}$ can be found by neglecting $z^{2}$ in $z^{2}-\gamma_{0}{ }^{2}$ of Eq. (3.2-18).

Provided $\omega_{0}{ }^{2}>\gamma_{0}{ }^{2}$, the growth rate will be determined by $z_{1}$, typically of the order $\frac{1}{\tau}$. The remaining roots will be high frequency oscillations that decay away with a similar time constant. If, however, $\gamma_{0}{ }^{2}>\omega_{0}{ }^{2}$, the growth rate will be predicted by Eq. (3.2-25). 


\subsection{APPLICATION TO TNS}

Predicting an accurate value for the growth rate in TNS with first wall included would be a difficult task due to the complicated geometry of the plasma and first wal1. An estimation using Eqs. (3.2-24) and (3.2-25) is possible, however, provided an effective wall radius can be chosen that is physically meaningful. Since the eddy currents have an approximate $\sin \theta$ distribution, a value $\rho_{w} \approx b^{-}$ can be used, where $b^{-}$is the wall radius at $\theta=\pi / 2$. The growth rate predicted would then be conservatively high since $b^{-}$is the position in the wall furthermost from the origin $\rho=0$. For a scrape-off zone of $0.2 \mathrm{~m}$, the value of $\rho_{\mathrm{W}} \approx \mathrm{b}^{-}=2.2 \mathrm{~m}$. Using a conductivity of stainless steel $\sigma=1.1 \times 10^{6} \Omega^{-1} \mathrm{~m}^{-1}$, a wall thickness of $12 \mathrm{~mm}, \gamma_{0}^{2}=1.28 \times 10^{11} \mathrm{~s}^{-2}$ (from Section 2.2), and the remaining parameters needed in Table 2.2-1, the values of the parameters $\omega_{0}^{2}$ and $\tau$ are

$$
\begin{gathered}
\omega_{0}^{2}=3.73 \times 10^{11} \mathrm{sec}^{-2} \\
\tau=0.018 \mathrm{sec}
\end{gathered}
$$

Since $\omega_{0}^{2}>\gamma_{0}^{2}, z_{1}$ predicts the growth rate

$$
\gamma=z_{1}=29 \mathrm{sec}^{-1}
$$

The high frequency damped oscillations are then

$$
z_{2,3}= \pm i\left(5 \times 10^{5}\right)-42 \mathrm{sec}^{-1}
$$

Since the current diffusion time $t_{s}=0.1 \mathrm{~ms}$ and $\left|\frac{1}{z_{2}, 3}\right|=2 \mathrm{~ms}$, the high frequency oscillations may be damped considerably. The predicted damping time is $\sim 24 \mathrm{~ms}$, so they should present no problem irregardless.

It is useful to see the tendency of the growth rate variation with the decay index even though it is a function of $B, B_{v}, I_{p}$, etc. Assuming all the other parameters can be held constant, the growth rate will increase drastically with $-n$ and appears to diverge at $\omega_{0}^{2}=\gamma_{0}{ }^{2}$. 
Figure 3.3-1 shows the variation of $z$ with $-n$. Approximations $(3.2-24)$ and (3.2-25) break down near $\omega_{0}=\gamma_{0}^{2}$ and cause this apparent divergence.

As has been mentioned earlier, the toroidal effect is of higher order and was not considered. However, it can be discussed qualitatively. At $15 \%$, the induced current has the form

$$
j_{W}=j_{0} \sin \theta(1+0.3 \cos \theta) \text {. }
$$

This indicate's the distribution of induced current is weighted to the outside of the torus in the direction of shifting magnetic axis for finite $B$.

To summarize the highlights of this section, we have seen that a dispersion relation of third order develops from the plasma equation of motion and the wall circuit equation. There will be one growth rate and two damped roots. If the wall is close enough, the growth rate will be considerably reduced from the value computed in Section 2 with no stabilizing mechanism. If it is too far removed, the growth rate will approach this value.

For TNS, this growth rate is $29 \mathrm{sec}^{-1}$ corresponding to a rising time of $34 \mathrm{~ms}$. The rising time computed with no stabilizing mechanism was found to be $2.8 \mu \mathrm{s}$. Thus, the wall will slow the instability by a factor of over $10^{4}$.

A growth rate of $30 \mathrm{sec}^{-1}$ is controllable by today's technology. However, the first wall in TNS may require transverse insulating gaps to allow the external fields to diffuse more rapidly into the vacuum vessel. The higher wall resistance would thereby increase the growth rate proportionally. For this reason, it is necessary to investigate the stabilizing properties of other external conductors. Since the EF coils are continuous in the toroidal direction, they may support the eddy currents required for slowing down the vertical instability. 


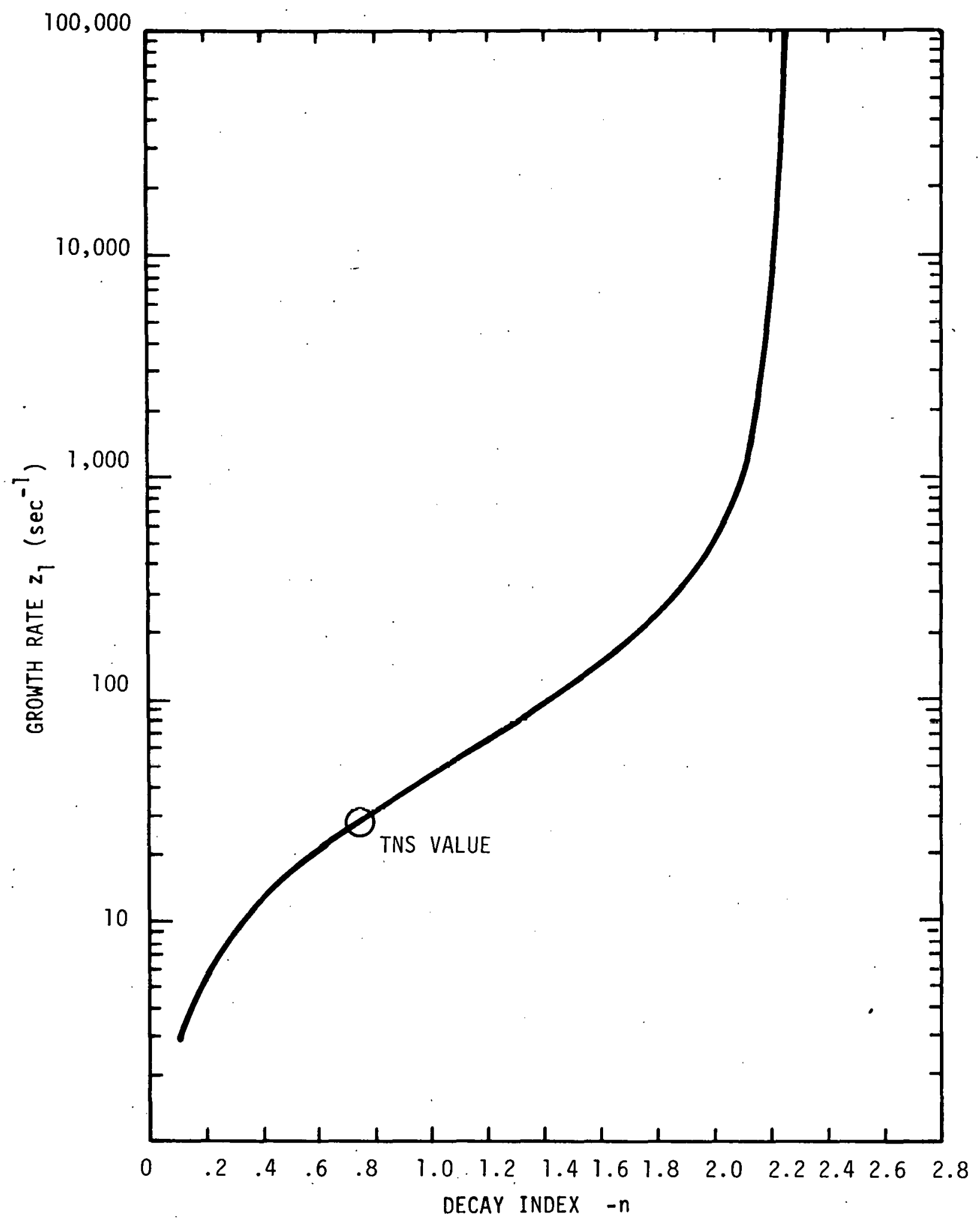

Figure 3.3-1 Growth Rate With Stabilizing Effect of First Wall VS Decay Index 


\subsection{STABILIZING EFFECT OF THE EQUILIBRIUM FIELD COILS ON THE VERTICAL INSTABILITY}

In this section we shall consider the effect of the equilibrium field coils on the growth rate of the vertical instability. This treatment is expected to describe the actual behavior of the plasma if the first wall is weak or contains transverse gaps that impede the flow of stabilizing eddy currents. It is assumed that steady state has been achieved and the $\mathrm{OH}$ coils are decoupled from the plasma .

An induced plasma skin current is found to lessen the growth rate by only a few percent when stabilized by the first wall, so for simplicity and conservatism, we shall neglect any induced plasma currents in this treatment. We shall also approximate the plasma as a current loop for simplicity, since finite plasma size did not appear in the growth rate equations. The fields due to current loops can be computed accurately by means of the elliptic integrals, so an exact solution that includes toroidal effects is possible for this discrete coil approach. The plasma equation of motion and the EF coil circuit equations are developed in much the same manner as they were for the resistive wall. It is shown that the coils above the equatorial plane must, by some method, be connected in parallel with those below the plane so the stabilizing eddy currents are free to flow. The directions and magnitudes of the coil currents thereby resembles the approximate $\sin \theta$ distribution found in the wall. Instead of one circuit equation, however, there will be $N$ circuit equations where $N$ is the number of EF coil pairs. By connecting coils above the equatorial plane in series and placing them in parallel with those below, the number of independent circuit equations can be reduced. Three configurations are studied in which 10,3 , and 1 independent circuit equations result.

The shell effect for each configuration is demonstrated by means of an eigenvalue equation for the induced coil currents. Varying the decay index permits the extraction of an effective $\omega_{0}^{2}$ and $\tau$ by fitting the numerical values of the growth rate to the shell formula $z_{1}$, given by $E q .(3.2-24)$. 


\subsection{DEVELOPMENT OF THE EQUATION OF MOTION AND THE EF COIL CIRCUIT EQUATIONS}

Consider the EF coil pair symmetrically located about the equatorial plane $z=0$. A current $I_{0}$ flows in the top and bottom coil in equilibrium. An induced current $I_{i}$ also flows when the plasma is perturbed $\xi \hat{z}$. Since this motion increases the flux in the top coil and decreases the flux in the bottom, the direction of the induced current $I_{j}$ is as shown for the parallel configuration (Figure 4.1-1). Note that $I_{i}$ is free to flow by bypassing the power supply and the top and bottom contributions do not cancel as they would in a series connection. The induced current gives rise to a radial field $(9)$

$$
b r_{i}=-\frac{\mu_{0} N_{i} I_{i} z_{i} g_{i}(K, E)}{2 \pi R_{p}\left[\left(r_{i}+R_{p}\right)^{2}+z_{i}^{2}\right]^{T / 2}}
$$

at the plasma radius $R_{p}$ where $N_{i}$ is the number of turns in the top coil, $r_{i}$ and $z_{i}$ define $i t s$ position, and $g_{i}(K, E)$ is a function of the complete elliptic integrals

$$
g_{i}(K, E)=-K\left(k_{i}{ }^{2}\right)+\frac{r_{i}{ }^{2}+R_{p}^{2}+z_{i}{ }^{2}}{\left(r_{i}-R_{p}\right)^{2}+z_{i}{ }^{2}} E\left(k_{i}{ }^{2}\right)
$$

with arguments

$$
k_{i}^{2}=\frac{4 r_{i} R_{p}}{\left(r_{i}+R_{p}\right)^{2}+z_{i}^{2}}
$$

Since $I_{i}$ flows in the opposite sense in the bottom coil, it will give rise to the same radial field on axis. Thus, the equation of motion of the plasma can be expressed

$$
\frac{d^{2} \xi}{d t^{2}}-\gamma_{0}^{2} \xi=-\frac{2 I p}{m} \sum_{i=1}^{N} b r_{i}
$$

Assuming a time dependence $e^{z t}$, and using matrix notation, this is expressible as

$$
\left(z^{2}-\gamma_{0}^{2}\right) \xi_{0}=-\frac{A^{t}}{\mu} I
$$




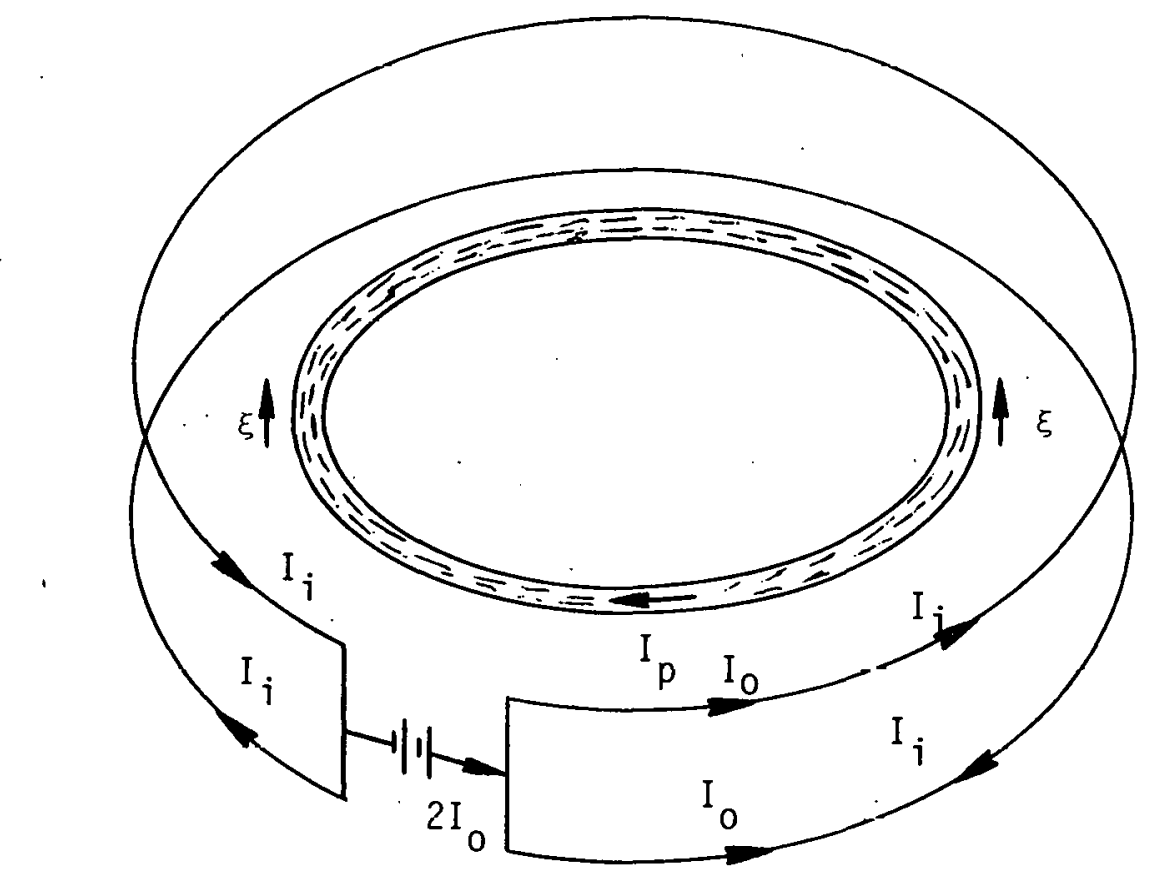

Figure 4.1-1. The Parallel EF Coil Pair Connection Showing Equilibrium Current $I_{0}$ and Induced Current $I_{j}$ Due to Vertical Motion of Plasma. The Plasma Current is Opposite in Direction to $I_{0}$ in all EF Coils Except the EF-D Coil. The Direction of $I_{j}$ is the same in all Coils for the Positive Perturbation $\xi \hat{z}$. In Practice, Each Top (Bottom) Coil will Consist of $\mathrm{N}_{j} \gg>1$ Turns and may be Connected in Series with. Other Top (Bottom) Coils and Collectively in Parallel with those Below (Above). 
where $\xi_{0}$ is the value of $\xi$ at $t=0, A^{t}$ is a row vector with components

$$
A_{i}=2 \pi R_{p} I_{p}\left(\frac{b_{r_{i}}}{I_{i}}\right)
$$

$I$ is a column vector with components $I_{j}$ at $t=0$, and $\mu$ is one-half the plasma mass

$$
\mu=\pi R_{p} m=M_{p} / 2 \text {. }
$$

A circuit equation for each coil pair can be developed in a manner that is analogous to the resistive wall, with the path defined in Figure 3.2-1 extended around the torus and including each of the $N_{i}$ turns in the top and bottom coils. In other words, the path around which $I_{j}$ flows in Figure 4.1-1 is taken. The emf's are as follows:

$$
\text { emf }{ }_{1 j}=-2 \sum_{i=1}^{N} M_{i j}^{-} \frac{d I}{d t}
$$

where $M_{i j}=M_{i j}-M_{i-j}$ is mutual inductance between two top coils $i$ and $j$ minus the mutual inductance between the top coil $i$ and the bottom coil $-j$. The factor of two arises by symmetry and the minus sign signifies the negative coupling between the top and bottom coils. The emf induced by the plasma motion can be expressed in the form

$$
e m f_{2 i}=-\frac{\partial}{\partial t} \oint_{i} \delta \underline{A}_{\phi} \cdot \underline{d l}
$$

or

$$
e m f_{2}=-2 \pi r_{i} N_{i} \frac{\partial}{\partial t} \delta A_{\phi i}
$$

where $\delta A_{\phi i}$ is the change in the vector potential of the plasma due to the perturbation $\xi \hat{z}$ evaluated at the position $r_{i}, z_{i}$. The last contribution is the IR drop

$$
\mathrm{emf}_{3_{i}}=-2 R_{\mathbf{i}} I_{\mathbf{i}}
$$


where $R_{i}$ is the resistance of the top (or bottom) coil. The three emf's are then summed to zero and result in $N$ circuit equations. The value of $\delta A_{\phi j}$, however, remains to be found. To find $\delta A_{\phi i}$, one uses $E q .(3.1-1)$ substituting $r \rightarrow r_{i}, z+z_{i}-\xi$ to define $A_{\phi i}$ at the perturbed position. The value of $k^{2}$ and $k$ are then changed as

$$
k^{2}+k_{i}^{2}\left(1+\frac{2 \xi z_{i}}{\left(R_{p}+r_{i}\right)^{2}+z_{i}^{2}}\right)
$$

and

$$
k \rightarrow k_{i}\left(1+\frac{\xi z_{j}}{\left(R_{p}+r_{i}\right)^{2}+z_{i}^{2}}\right) \equiv k_{i}+\delta k_{i}
$$

to first order in $\xi$. The elliptic integrals can be expanded in a Taylor series to order $\delta k_{i}$ making use of the properties of their derivatives

$$
\frac{\partial K\left(k_{i}^{2}\right)}{\partial k_{i}}=\frac{E\left(k_{i}^{2}\right)}{k_{i}\left(1-k_{i}^{2}\right)}-\frac{K\left(k_{i}^{2}\right)}{k_{i}} \text {. }
$$

and

$$
\frac{\partial E\left(k_{i}^{2}\right)}{\partial k_{i}}=\frac{E\left(k_{i}^{2}\right)}{k_{i}}-\frac{k\left(k_{i}^{2}\right)}{k_{i}}
$$

It can then be shown that $\delta A_{\phi i}$ takes the form:

$$
\delta A_{\phi i}=\frac{\mu_{0} I_{p} z_{i} \xi}{2 \cdot r_{i}\left[\left(R_{p}+r_{i}\right)^{2}+z_{i}{ }^{2}\right]^{T / 2}} g_{i}(K, E)
$$

Substituting this expression into Eq. (4.1-10) and defining $I_{p}$ in the negative sense,

$$
e_{2 i}=2 A_{i} \frac{d \xi}{d t}
$$

where $A_{i}$ is given by Eq. (4.1-6) 
Combining all the emf's and dividing by two, the circuit equations become

$$
\sum_{j=1}^{N} M_{i j}^{j} \frac{d I_{i}}{d t}+R_{i} I_{i}=A_{i} \frac{d \xi}{d t}
$$

which can be reexpressed in matrix notation assuming a time dependence $e^{z t}$,

$$
\left(z M^{\prime}+R\right) I=A z \xi_{0}
$$

where $M-$ and $R$ are $N \times N$ matricies with the following components and characteristics:

$$
M_{i j}=M_{i j}-M_{i-j}
$$

which is a positive definite symmetric matrix and

$$
R_{i j}=R_{i} \delta_{i j}
$$

is a positive diagonal matrix. A is an. $\mathrm{N}$ component column vector whose transpose appears in the equation of motion, Eq. (4.1-5)

$$
\left(z^{2}-\gamma_{0}^{2}\right) \xi_{0}=\frac{-A}{\mu} I
$$

It is interesting to note that the "cause and effect" vectors (i.e., A and $A^{t}$ ) have the same components. Since $\mu=M_{p} / 2$ it might appear that the effect is. twice as strong, however, Eq. $(4.1-19)$ applies to either the top or bottom. coil since the factor of two has been cancelled.

\section{i3}

There are two approaches one can use to eliminate I and $\xi_{0}$ from Eqs. (4.1-5) and (4.1-19) to get a dispersion relation for $z$. Eliminating $I$ first and then $\xi_{0} g$ ives

$$
z^{2}=\gamma_{0}^{2}-\frac{z A^{t}}{\mu}(z M-+R)^{-1} A
$$

where $\left(2 M^{-}+R\right)^{-1}$ is the inverse of $\left(2 M^{-}+R\right)$. For general matricies, the inverse would be difficult to find since $R$ and $M$ - would not be simultaneously 
diagonalizable unless they commute. As they appear here, $R$ and $M^{-}$do not commute either. A way has been devised in another work to deal with this problem and cast the right hand side of Eq. $(4.1-20)$ into a workable form ${ }^{(12)}$. Since $R$ is diagonal, $R^{-1 / 2}$ and $R^{1 / 2}$ can be easily defined. Using properties of the inverse, we may write

$$
A^{t}(z M-+R)^{-1} A=\left(R^{-1 / 2} A\right)^{t}\left[z\left(R^{-1 / 2} M-R^{-1 / 2}\right)+1\right]^{-1}\left(R^{-1 / 2} A\right)
$$

where $\mathrm{l}$ is the $\mathrm{N} \times \mathrm{N}$ identity matrix.

It is easily shown that $R^{-1 / 2} M-R^{-1 / 2}$ remains positive, definite, and symmetric. Therefore, it can be diagonalized by an orthogonal matrix $U$ that yields positive components

$$
U\left[R^{-1 / 2} M-R^{-1 / 2}\right] U^{t}=\lambda^{-1}
$$

where

$$
\lambda_{i j}^{-1}=\frac{1}{\lambda_{i}} \delta_{i j}, \lambda_{i}>0
$$

Redefining $\tilde{A}=U R^{-1 / 2} \mathrm{~A}$, Eq. $(4.1-21)$ becomes

$$
A^{t}\left(z M^{-}+R\right)^{-1} A=\tilde{A}^{t}\left(z \lambda^{-1}+1\right)^{-1} \tilde{A}
$$

and Eq. $(4 \cdot 1-20)$ can be rewritten as

$$
r_{0}^{2}=z^{2}+\sum_{j=1}^{N} \frac{c_{i} z}{z+\lambda_{i}}
$$

where

$$
c_{i}=\frac{\lambda_{i} \tilde{A}_{i}^{2}}{\mu}>0
$$

Equation $(5.1-19)$ bears a striking resemblance to the in-hour equation in reactor kinetics $(13)$. The quantities $\gamma_{0}{ }^{2}, c_{i}$, and $\lambda_{i}$, correspond to reactivity, delayed neutron fraction of the $i$-th group, and delayed neutron lifetime in the $i$-th group. The reactor period would be $1 / z$. The prompt neutron contribution $\ell z$ that 
appears in the in-hour equation $(\bar{l}=$ prompt neutron lifetime) is replaced by the inertial term $z^{2}$ in Eq. (4.1-25). Since the $\lambda_{j}^{-1}$ 's are related to the L/R times of the coils, they are (and have been demonstrated to be) positive. Thus, there are $N$ poles at $z_{i}=\lambda_{i}$. Graphing the right-hand side of Eq. (4.1-25), the $z$ axis is cut at $z=0$ and behaves asymptotically as $z^{2}$ for large $z$. At large negative $z, z^{2}$ again dominates. The poles confine the other branches as shown in Figure 4.1-2.

As is graphically demonstrated with $N=5$ for $\gamma_{0}{ }^{2}>0$, there will be one unstable root $z_{1}$. For moderate values of $\gamma_{0}{ }^{2}, z_{1}$ will intersect near the origin and there will be $N-1$ negative real roots following. The two remaining roots will be damped complex conjugate pairs. For large $\gamma_{0}^{2}, z$ will be highly unstable, intersecting near the portion of the graph behaving as $z^{2}$ asymptotically. The $\mathrm{N}-1$ negative real roots found before almost remain fixed. Another negative root then appears following these, and finally the last highly negative root appears which is almost a mirror image of $z_{1}$. In summary, there are $N+1$ damped roots and one highly unstable root. For negative $\gamma_{0}^{2}$, there are $N$ damped roots and two damped conjugate roots. It is easy to show that these observations are expected for $N=1$ by examination of the shell roots given in Eqs. $(3.2-24)$ and $(3.2-25)$.

For moderate values of $\gamma_{0}^{2}$, the effect of $z^{2}$ is not felt and the growth rate $\mathrm{z}_{1}$ and $\mathrm{N}-1$ damped roots can be found by neglecting $\mathrm{z}^{2}$ in Eq. $(4 \cdot 1-5)$. The two damped oscillatory pairs, however, would not be recovered. For large $\gamma_{0}^{2}$, one would find $N$ damped roots by neglecting $z^{2}$, but the principle roots of concern, $z \sim \pm \gamma_{0}$ would not be found.

With this word of caution, we drop $z^{2}$ in Eq. (4.1-5). Eliminating $\gamma_{0}$ then gives

$$
\left(z M^{-}+R\right) I=\frac{z A A^{t} I}{\mu \gamma_{C}{ }^{2}}
$$

dividing by $z$ and rearranging

$$
\left(M--\frac{A A^{t}}{\mu \gamma_{0}^{2}}\right) I=-\frac{R}{2} I
$$




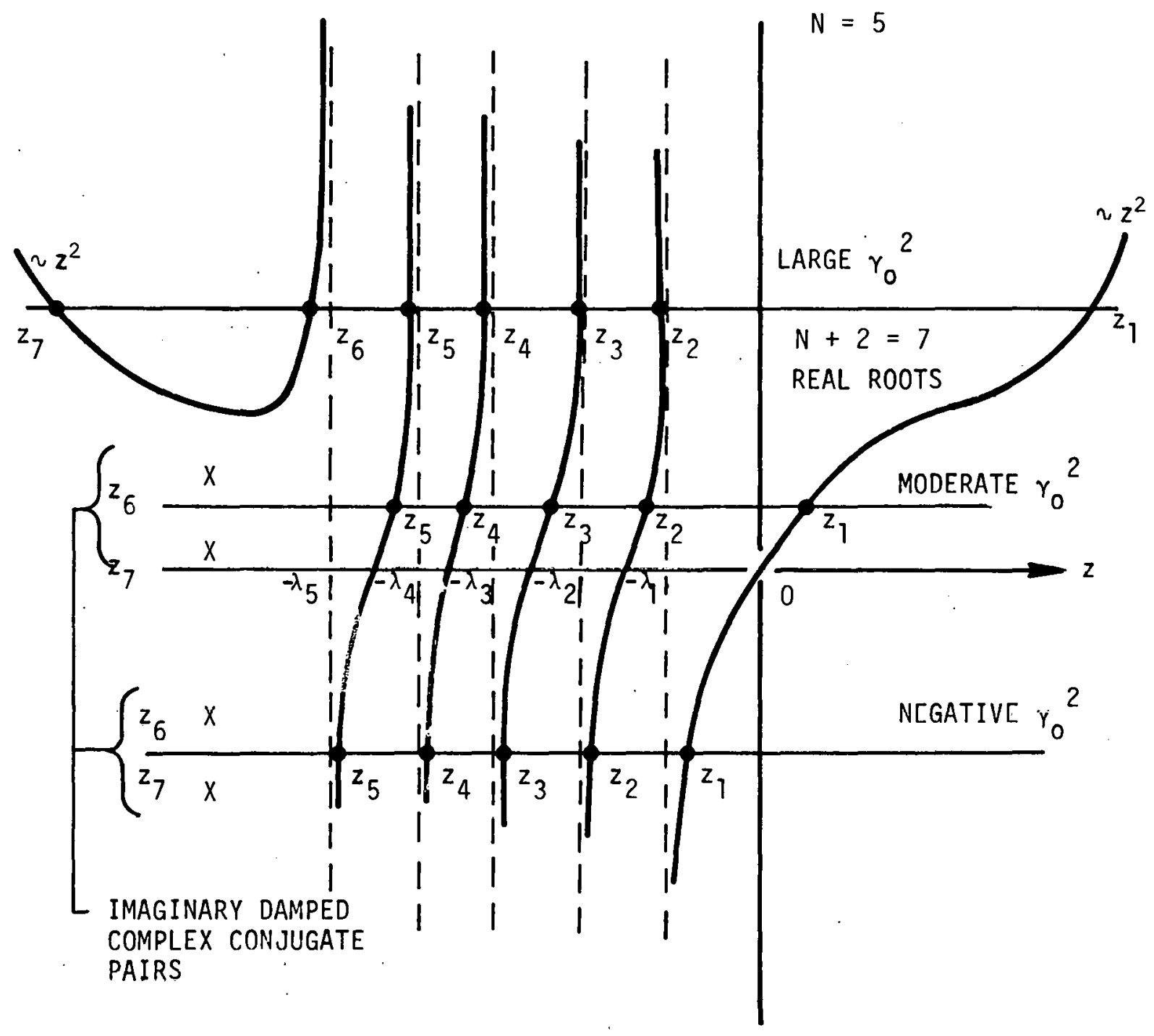

Figure 4:1-2. Graphical Analysis of Growth Rate for EF Coil Configurations. Sketch of 4.1-25 for $N=5$. 
which can be rewritten in the form of an eigenvalue equation for the induced coill current amplitudes.

$$
H I=-\frac{1}{2} I
$$

where the components of $H$ are given by:

$$
H_{i, j}=\frac{1}{R_{i}}\left(M_{i j}-\frac{A_{i} A_{j}}{\mu \gamma_{0}^{2}}\right)
$$

Equation (4.1-29) can be solved numerically for the $N$ eigenvalues $-1 / z$. The eigenvectors give the relative coil current amplitudes. Normalization of the coil amplitudes is then possible using either Eq. (4.1-19) or (4.1-5) with $\xi_{0}$ specified.

It is relatively simple to include the effect of connecting two or more coils above the plane in a series and then connecting them in parallel to those below. The value of $\mathrm{I}_{i}$ in these coils so connected is fixed and one can add their curcuit equations together under this constraint to obtain a fewer number of independent circuit equations. The limiting case is the all series-parallel connection. Here, one has the same current in all the coils and all the matricies and vectors become scalars. Then one has

$$
\begin{aligned}
& M^{-}=\sum_{i, j=1}^{N} M_{i j} \\
& A=A^{t}=\sum_{i=1}^{N} A_{i} \\
& R=\sum_{i=1}^{N} R_{i}
\end{aligned}
$$

and Eq. $(4.7-29)$ can then be solved directly giving

$$
z=\frac{1}{(M-/ R)} \frac{\gamma_{0}^{2}}{\left(A^{2} / \mu M-\right)-\gamma_{0}^{2}}
$$


which leads to the effective $\omega_{0}^{2}=A^{2} / \mu M$ - as used in the shell formula. The other parameter was perhaps obvious. Effective $\omega_{0}^{2}$ and $\tau$ for other configurations of higher order can be fitted to the solution $z_{1}$, given by Eq. (3.2-25) by varying $\gamma_{0}^{2}$ and solving numerically. This will be demonstrated in the TNS application.

\subsection{APPLICATION TO TNS}

Using the realistic inductance and resistance matricies, the eigenvalue equation (4.1-29) is solved for the induced coil currents amplitudes I and the growth rate and damped roots $z$. Three configurations are examined for copper EF coils (resistivity $=1.72 \mu \Omega \mathrm{cm}$ ).

Configuration I uses the ten parallel EF coils pairs with each pair having its own independent power supply. The $10 \times 10$ inductance matrix $M^{-}$is given in Table 4.2-1. The values of the resistance matrix $R$ and the vector $A$ (for $1_{p}=$ 6.1 MA) with the positions $\left(r_{i}, z_{i}\right)$ and the coil turns $N_{i}$ are given in Table 4.2-2.

In Configuration II, the inside ( 1 thru 4), divertor like (5) and outside ( 6 thru 10) coils are placed in the series-parallel connection. By adding the circuit equations together, the contracted inductance matrix (also given in Table 4.2-1) results. This matrix can be easily compiled from the previous one by adding up all the inductances in each of the nine block matricies highlighted in the $10 \times 10$ matrix. The components of $R$ and $A$, contracted in the same manner, are given in 4.2-2 below Configuration I's values. Since each coil within these three groups requires the same equilibrium current as the others in the group, this configuration is practical as well as economical in that only three power supplies are required.

Conflguration III was the all series-parallel connection, with $M^{-}, A$, and $R$ given by Eqs. (4.1-31) thru $(4.1-33)$. The growth rate is given by Eq. (4.1-34) and there are no real damped roots until $A^{2} / \mu M^{-}<\gamma_{0}{ }^{2}$. This configuration is not practical in that the I, $D$, and 0 equilibrium currents are all different and are brought to their steady state values at different rates, but it does 
TABLE $4.2-1$

INDUCTANCE MATRIX $M-\left(10^{2} H\right)$ FOR TNS REFERENCE FOR CONFIGURATION I

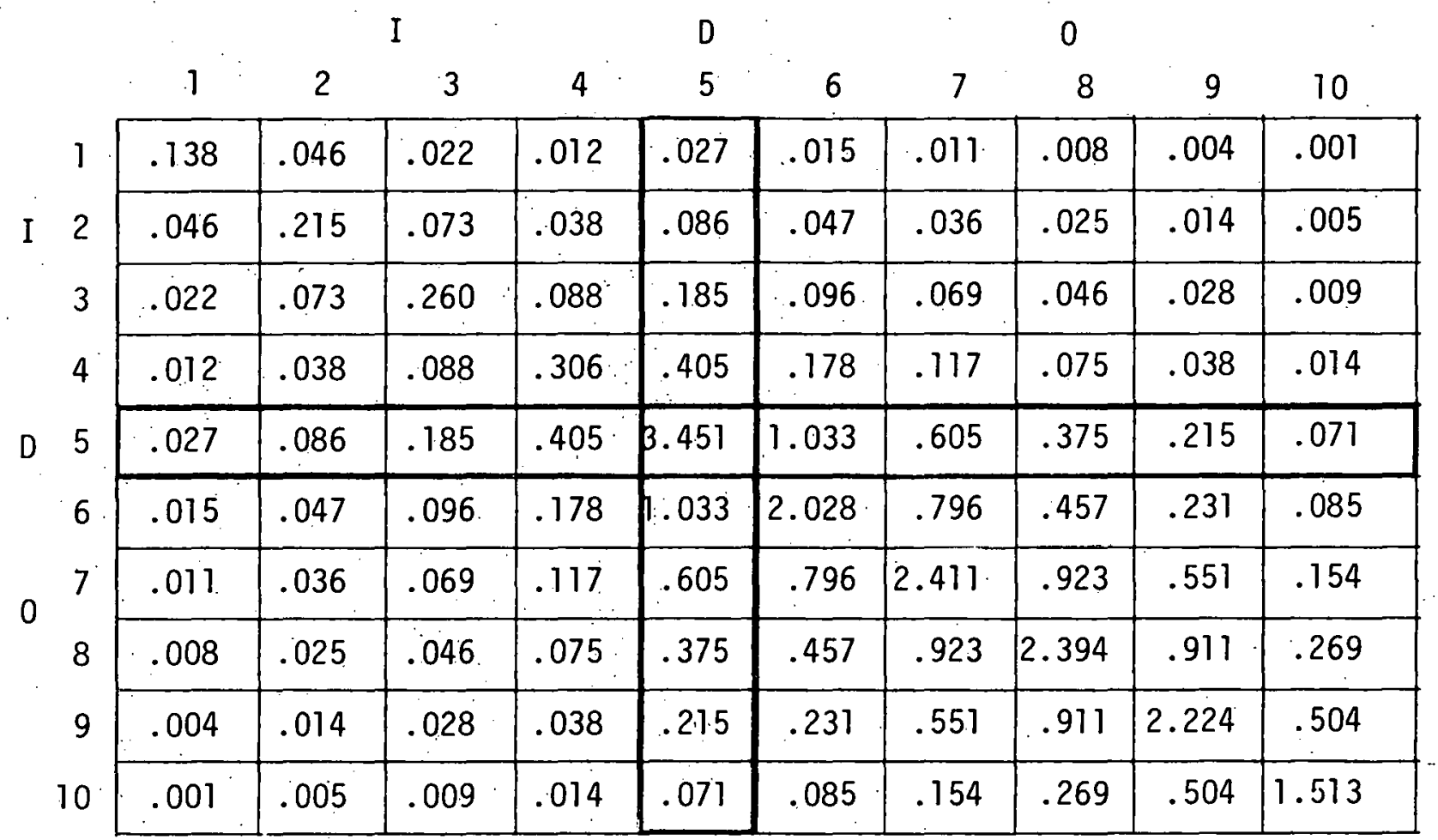

CONTRACTED MATRIX FOR CONFIGURATION II

$\left(\times 10^{2} \mathrm{H}\right)$

\begin{tabular}{|r|r|r|}
\hline 1.477 & .703 & .836 \\
\hline .703 & 3.451 & 2.299 \\
\hline .836 & 2.299 & 20.332 \\
\hline
\end{tabular}

$M^{-}=.329 \mathrm{H}$

Scalar matrix for Configuration III 
TABLE $4.2-2$

DATA FOR EF COIL PAIRS IN CONFIGURATION I

\begin{tabular}{|c|c|c|c|c|c|c|}
\hline & $\begin{array}{l}\text { COIL } \\
\text { PAIR }\end{array}$ & $A_{i}\left(\frac{N t}{A m p}\right)$ & $N_{i}$ (TURNS) & $R_{i}$ (OHMS) & $r_{i}(m)$ & $z_{i}(m)$ \\
\hline & 7 & 20.6 & 13.4 & .00428 & 3.15 & .21 \\
\hline & 2 & 58.7 & 13.4 & .00437 & 3.17 & .65 \\
\hline & 3 & 90.9 & 13.4 & .00442 & 3.25 & 1.26 \\
\hline & 4 & 107. & 13.4 & .00477 & 3.51 & 2.02 \\
\hline & 5 & 396 , & 44.8 & $\cdot .0182$ & 4.00 & 2.59 \\
\hline & 6 & 360. & 29.8 & .0148 & 4.90 & 2.55 \\
\hline & 7 & 446. & 29.8 & .0174 & 5.75 & 2.10 \\
\hline & 8 & 438. & 29.8 & .0190 & 6.29 & 1.51 \\
\hline & 9 & 376. & 29.8 & .0200 & 6.63 & .95 \\
\hline & 10 & 117. & 29.8 & .0206 & 6.82 & .33 \\
\hline
\end{tabular}

CONTRACTED VALUES FOR

CONFIGURATION II

\begin{tabular}{|c|c|}
\hline$A_{i}\left(\frac{N t}{A m p}\right)$ & $R_{i}$ (OHMS) \\
\hline 227. & .0178 \\
396. & .0182 \\
1677. & .0918 \\
\hline
\end{tabular}

$A=2300 . \mathrm{Nt} /$ Amp.

$R=.128$ ohms

VALUES FOR CONFIGURATION III 


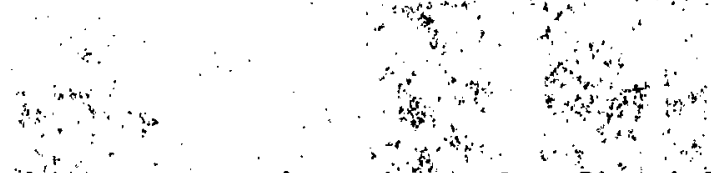

provide a comparison base for I and II when investigating the effect of the induced currents.

Using $\gamma_{0}^{2}=-n\left(1.68 \times 10^{11}\right)$ sec and $\mu=M_{p} / 2=6.47 \times 10^{-5} \mathrm{~kg}$, the matrix Eq. $(4.1-29)$ was solvedinumerically. There is one positive eigenvalue (growith rate) and $K-1(K=10,3$, or 1$)$ negative eigenvalues for each of the three configurations until the decay index exceeds some critical value. For configuration $I$ this critical value is roughly ${ }_{c r}=-1.66$. Due to the constraint on the currents in II and III, the critical value is lowered in magnitude to $n_{c r}=-1.57$ and -1.54 , respectively. The growth rates are plotted as a function of the decay index in Figure 4.21 where all the other plasma parameters are assumed constant. The growth rates for II and III are almost equal when $-n$ is less than 1. Below $n=10$ they differ only in the fourth significant figure, with II being the more stable. In Configuration I, the induced currents are not constrained and free to adjust to their approximate $\sin \theta$ distribution. As expected, the growth rates are; slightiy lower than in II and III.

Using $n=-0.8$ for TNS, the three growth rates are

$$
\begin{aligned}
\gamma & =3533 \mathrm{sec}^{-1} \quad \text { For I } \\
\gamma & =4185 \mathrm{sec}^{-1} \text { For II } \\
\gamma \gamma & =4188 \mathrm{sec}^{-1} \text { For III }
\end{aligned}
$$

which correspond to rising times of $2.83,2.39$, and $2.39 \mathrm{sec}$, respectively. We note considerable improvement here over first wall stabilization (34 ms) and no stabilization $(2.8 \mathrm{us})$ by two and six orders of magnitude, respectively.

The effect of resistance (or resistivity) can be noted from the form of the $H_{i j}$ components given in Eq. $(4.1-30)$, the growth rate being proportioned to the coil resistivity. Thus, superconducting coils will provide neutral stabilization for $-n<-n_{\mathrm{cr}}$.

The coils have many features similiar to the resistive wall and it is possible to fit the analytic shell growth rate $z_{1}$ in Eq.: $(3.2-24)$ to the numerical data and thereby define an effective $\omega_{0}^{2}$ and $\tau$ for the coils. Configuration III, 


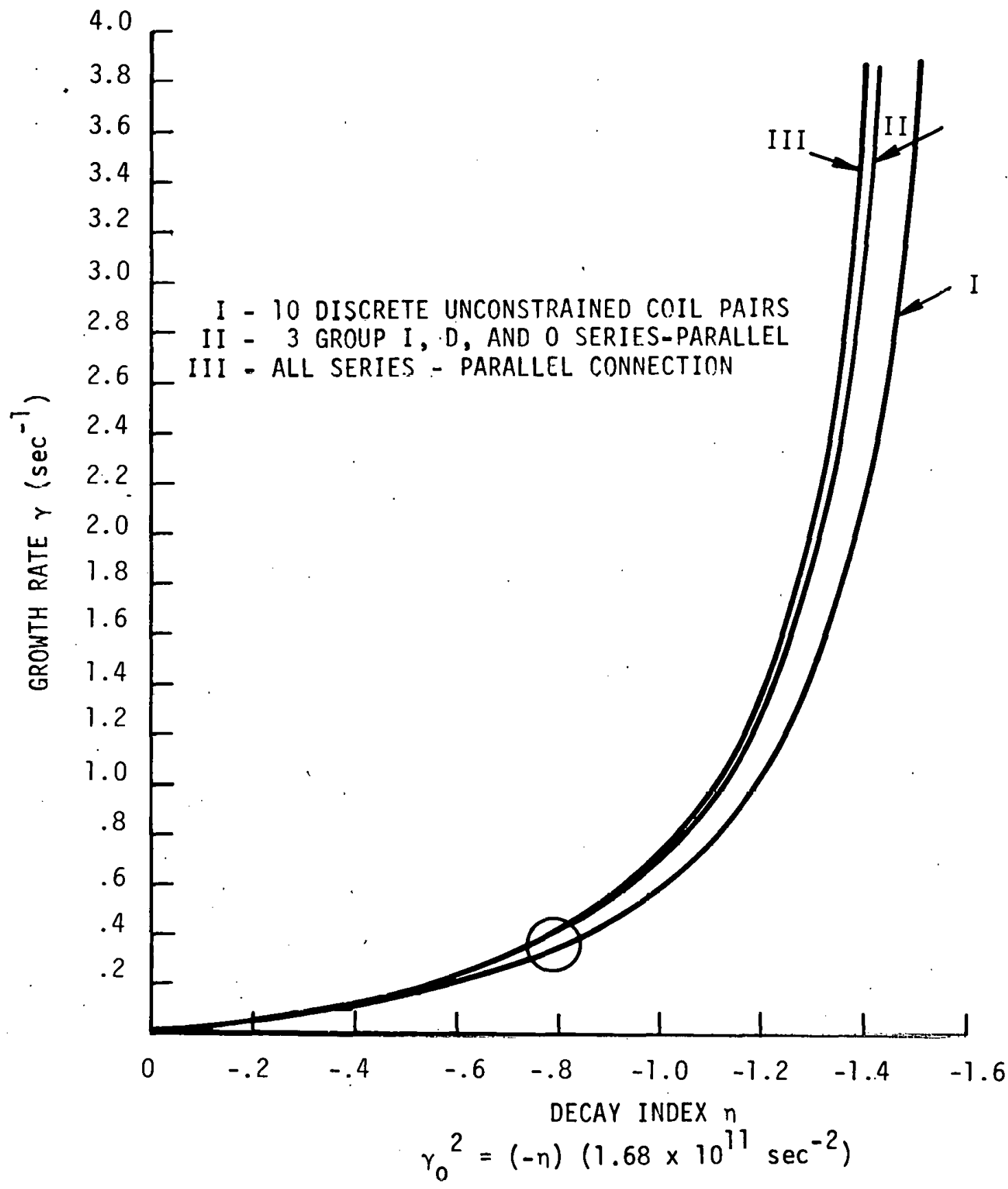

Figure 4.2-1. Growth Rate of Vertical Instability vs Decay Index for Different Parallel EF Coil Configurations when Other Plasma Parameters are Held Constant. 
of course, already obeys this formula and its values can be fitted exactly. The result is an effective $\omega_{0}^{2}=2.59 \times 10^{11} \mathrm{sec}^{-2}$ and $\tau=2.57 \mathrm{sec}$.

For Configuration I, effective $\omega_{0}^{2}=2.77 \times 10^{11} \mathrm{sec}^{-2}$ and $\tau=2.70 \mathrm{sec}$ provide agreement between the analytic expression and the numerical data to less than $5 \%$ deviation over the decay index range $0<-n<1.6$. For Configuration II, the effective $\omega_{0}^{2}=2.64 \times 10^{11} \mathrm{sec}^{-2}$ and $\tau=2.50 \mathrm{sec}$ gives agreement to $5 \%$ over the range $0<-n<1.4$.

Having defined the effective $\omega_{0}^{2}$ and $\tau$ for the three configurations, it is possible to estimate the high frequency damped roots for TNS that have been neglected in this treatment. Using $n \simeq-0.8, z_{1} \simeq 0.4 \mathrm{sec}^{-1}, \omega_{0}^{2}=2.6 \times 10^{11}$ $\mathrm{sec}^{-2}$, and $\tau=2.6 \mathrm{sec}$, Eq. $(3.2-2.5)$ gives

$$
z_{2,3} \simeq \pm i\left(3.5 \times 10^{5}\right)-0.4 \mathrm{sec}^{-1}
$$

Since these roots are damped and may be artifacts, they should present no problem.

The eigenvectors of the matrix equation give the relative current amplitudes in the EF coils. When normalized to $\xi_{0}=0.1 \mathrm{~m}$, the induced currents contribute an inward stabilizing radial field of 91 gauss at $r=R_{p}, z=0$. The currents of the positive growth rate are all of the same sign (i.e., in the direction $+\hat{e}_{\phi}$ in the top coils) but of mixed signs for the $k-1$ damped roots $z<0$. The induced EF coil currents for $z_{1}$, TNS Configuration I $(n=-0.8)$ are given in Figure 2.3-1. The currents appear somewhat as the sin $\theta$ distribution that gave rise to the uniform radial field for first wall stabilization. The induced field at $r=R_{p}, z=0.5 \mathrm{~m}$ was examined to obtain some idea of the field uniformity. It was found that an inward radial field of 89 gauss and a vertical field of 15 gauss exists at this point. This radial field is only $2 \%$ lower than the induced field on axis and the vertical field is less than $0.3 \%$ of the equilibrium vertical field. Thus, it appears that the radial field is rather uniform or at least smoothly varying over the plasma cross section and the induced vertical field component does not significantly disturb the vertical field required for horizontal equilibrium. 
As has been discussed previously, the effective conducting shell approximation is considered to be reasonable and we rely on it to simplify the calculations for the feedback control system discussed in the next section. 


\subsection{FEEDBACK CONTROL OF THE VERTICAL INSTABILITY}

The discrete EF coils. Were found to slow the vertical instability in TNS to rising times on the order of 3 seconds. Since the discharge time in TNS will be about 30 seconds, some type of feedback control will be required to assure plasma stability for the pulse duration.

Having demonstrated an equivalence between the EF coils and a resistive shell, we shall rely on this property to simplify the control theory when a more exact solution is not possible. This shell-like approximation is applicable to Emmert's approach (14) where instantaneous and pure time-delayed feedback is considered. Hugill and Gibson $(15)$, on the other hand, use a controller voltage determined by both the plasma position and the detection and amplifier time constant. Their analysis is by circuit theory in which both passive and active agents have their own (collective) resistances and inductances. A similarity between the two approaches is demonstrated by comparing the gain requirements and the delay and controller time constants.

A brief summary of different optical and magnetic probe detectors is given. $X$-ray detectors appear to be fast responding and capable of sufficient spatial resolution provided a system of several detectors is used. Present state-ofthe-art magnetic probes may be adequate to detect $\xi_{0}<0.1 \mathrm{~m}$, however, sensitivity could be reduced if these are placed outside the vacuum vessel.

A conceptual design for vertical feedback control in TNS is then presented. This system uses three separate control windings located near the $0-5,0-6$, and 0-7 EF coils. These control coils may be wound in the same casings as the EF coils but separately connected, however. The peak power requirements to vertically control the plasma within $\pm 0.1 \mathrm{~m}$ (assuming adequate detection is possible) is less than ? MW for a 6.1 MA plasma current and a delay time of $1 \mathrm{sec}$. A peak power approximately 10 times this value would be needed if EF coil currents provided the active control instead of the separate windings. 
5.1 CONTROL THEORY FOR FEEDBACK CONTROL OF THE VERTICAL INSTABILITY

Since the EF coils behaved collectively like a shell, we shall assume that they can be treated as if $R, M^{-}$, and $A=A^{t}$ are scalars and avoid the matrix nature of these quantities. Let us assume that

$$
B_{r}^{f . b .}=-G \xi\left(t-t_{d}\right)
$$

where $t_{d}$ is the time delay for the control system and $G$ is the controller gain $(T / m)$. The equation of motion for the plasma (see Eq. $(4.1-4)$ and $(4.1-5)$ ) is then modified to include the $I_{p} \times{ }_{r}^{f}$.b. feedback force as

$$
\frac{d^{2} \xi(t)}{d t^{2}}-\gamma_{0}^{2} \xi(t)=\frac{-A I_{c}}{\mu}-\frac{I_{p}^{G}}{m} \xi\left(t-t_{d}\right)
$$

where $I_{c}$ is the effective EF coil current and $\mu=\pi R_{p} m$ is half the plasma mass. The circuit Eqs. (4.1-18) or (4.1-19) will contain an emf due to the coupling between the feedback field and the induced coil current $I_{C}$. The emf due to the feedback control coils can be written as

$$
\text { emf } f \cdot b .=4 \pi R_{p} \rho_{c} \frac{d}{d t} B_{r}^{f . b .}
$$

where $\rho_{C}$ is the effective radius of the shell for the EF coils. Dividing by two and recalling Eqs. $(5.1-1)$ and $(4.1-18)$, the modified circuit equation becomes

$$
R I_{c}(t)+M-\frac{d I_{c}(t)}{d t}=A \frac{d \xi(t)}{d t}-\left(2 \pi R_{p} \rho_{c}\right) G \frac{d}{d t} \xi\left(t-t_{d}\right)
$$

Defining $\tilde{f}(t)$ as the LaPlace transform of $f(t)$, Eqs. $(5.1-2)$ and $(5.1-4)$ can be rewritten as

$$
s^{2} \tilde{\xi}-\dot{\xi}(0)-s \xi(0)-\gamma_{0}^{2} \tilde{\xi}=-\frac{A}{\mu} \tilde{I}_{c}-\frac{I_{p} G}{m} \tilde{\xi} e^{-s t_{d}}
$$

and

$$
\left(R+M^{-} s\right) \tilde{I}_{c}-M \cdot I_{c}(0)=A[s \tilde{\xi}-\xi(0)]-2 \pi R{ }_{p} \rho_{c} G e^{-s t_{d}}[s \tilde{\xi}-\xi(0)] .
$$


Neglecting initial conditions $\xi(0)=\dot{\xi}(0)=I_{c}(0)=0$, the dispersion relation becomes

$$
s^{3}+\frac{1}{\tau} s^{2}+\left(\omega_{0}{ }^{2}-\gamma_{0}{ }^{2}\right) s+\frac{1}{\tau}\left(\frac{I p}{m}\right) e^{-s t_{d}}-\frac{\gamma_{0}{ }^{2}}{\tau}=0
$$

In the 7 imit $t_{d} \rightarrow 0$, it is easy to see from the Roth array and Eq. (3.2-22) that the stability condition for Eq. (5.7-7) becomes

$$
G>\frac{m r_{0}^{2}}{I_{p}}=-\frac{n B_{z}}{R_{p}}
$$

and the roots are given by Eqs. $(3.2-24)$ and $(3,2-25)$ with $\frac{I_{p} G}{m}-\gamma_{0}{ }^{2}$ replacing $-\gamma_{0}{ }^{\prime}$ in the numerator of Eq. $(3.2-24)$. Thus,

$$
s_{1}=-\frac{\left(\frac{I p}{m} G-\gamma_{0}{ }^{2}\right)}{\left(\omega_{0}{ }^{2}-\gamma_{0}{ }^{2}\right)}
$$

and

$$
s_{2,3}= \pm i\left(\omega_{0}^{2}+-\gamma_{0}{ }^{2}\right)^{\frac{1}{2}}-\frac{1}{2}\left(s_{1}+\frac{1}{\tau}\right)
$$

describe the long term plasma behavior $\xi \mathrm{e}^{\mathrm{st}}$ in the 1 imit of instantaneous feedback $t_{d} \rightarrow 0$.

Since we are interested in the low frequency behavior (i.e., $s_{2,3}$ should be stabilized by the wall) we shall neglect the first two terms of Eq. (5.1-7) obtaining

$$
\frac{s}{z_{1}}=1-g e^{-s t_{d}}
$$

where $z_{1}$ is the growth rate of Eq. (3.2-24) or the growth rate with EF coil stabilization obtained numerically and

$$
g=\frac{I P^{G}}{m Y_{0}^{2}}
$$


is the relative gain of the feedback control system. Emmert ${ }^{(14)}$ has shown; using Nyquist diagrams, that the conditions of stability $\left(R_{e} s<0\right)$ for $E q$. $(5.1-11)$ are

$$
g>1
$$

and

$$
t_{d}<\frac{1}{z_{1}} \frac{1}{\sqrt{g^{2}-1}} \cos ^{-1}\left(\frac{1}{g}\right)
$$

The result $g>1$ is expected since it is a restatement of Eq. (5.1-8) when $t_{d} \rightarrow 0$. The delay time must always be less than the rising time $1 / z$, and can approach. this value as $g \rightarrow r$. This is rather unexpected since a higher gain requires a smaller delay time.

A more restrictive condition on $t_{d}$ applies if the transient response is considered. If given an initial displacement $\xi_{0}$, the stabilized plasma will be reversed at $t=t_{d}$ if the restoring force exceeds the driving force on the plasma. This condition is satisfied if

$$
\text { g. } \xi_{0}>\xi_{0} e^{z} t^{t}
$$

or

$$
t_{d}<\frac{1}{z_{1}} \ln (g)
$$

This pure growth and decay, however, does not occur at large gains and the condition of Eq. (5.1-14) should be met first in order to insure stability.

An attempt to optimize the power requirements for the feedback system has also been outlined by Emmert. Since a jump in $B_{r}^{f . b}$. would imply infinite power, a linear rise is assumed between $t=0$ and $t=t_{d}$. By matching the (magnetic) power required at $t_{d}$ and $2 t_{d}$, an optimum condition

$$
z_{1} \cdot t_{d} e^{2 \cdot z_{1} t_{d}}=1
$$

or

$$
t_{d}=\frac{.426}{z_{1}}
$$


is found". The gain requirement for transient stability is then

$$
g \geq 1.537
$$

by Eq. $(5.1-15)$. Since $g=1.537$ gives $t_{d}<\frac{.74}{z_{1}}$ by Eq. $(5.1-14)$, a stable solution is then assured for long term response also.

The maximum excursion of the plasma with $t_{d}$ given by Eq. $(5.1-16)$ will be $1.54 \xi_{0}$, and the power required to supply the change in the magnetic field will be

$$
P_{\text {mag }}=\frac{2}{t_{d}} G^{2} \xi_{0}^{2} U
$$

where $U$ is the stored magnetic energy per $\left(B_{r}^{f} \cdot b \cdot\right)^{2}$. The $I^{2} R$ loss in the controller must also be accounted for to determine the total power requirement.

Hugill and Gibson (15) approach this control problem in much the same manner as Emmert but express their circuit equations and equation of motion in terms of the shell and control windings inductances and resistances entirely. The controller voltage is given by

$$
t_{c} \frac{d V}{d t}+V=\frac{-\mu_{0} \pi R_{p} R_{3} K I_{p} \xi}{2 \rho_{w} M_{23}}
$$

where $V$ is the applied controller voltage, $R_{3}$ is the controller output resis tance, $K$ is a nondimensional gain of the servo-loop, $M_{23}$ is the mutual inductance between the wall (or in our case the EF coils) and the controller, and $t_{c}$ is the time constant of the detection and amplifier circuits.

The additional circuit equation for the controller and the presence of $V$ plus its derivative in Eq. (5.1-19) results in a fifth order dispersion relation for $z$ for $\xi \sim e^{z t}$. Neglecting the high frequency roots $\left(\left|z^{2}\right| \ll\left|\gamma_{0}{ }^{2}\right|\right)$, a third order equation develops

$$
C z^{3}+D z^{2}+E z+F=0
$$


where

$$
\begin{aligned}
& c=t_{c} t_{2}{ }^{2} \omega_{0}^{2} \\
& D=\left(t_{c} t_{1}+t_{2}{ }^{2}\right) \omega_{0}^{2} \\
& E=t_{1} \omega_{0}^{2}-t_{c} \gamma_{0}{ }^{2} \\
& F=k \omega_{0}^{2}-r_{0}{ }^{2}
\end{aligned}
$$

and $t_{1}$ and $t_{2}$ are defined by

$$
\begin{aligned}
& t_{1} \omega_{0}^{2}=\tau\left(\omega_{0}^{2}-\gamma_{0}^{2}\right)+t_{2}\left(k^{2} \omega_{0}^{2}-\gamma_{0}^{2}\right) \\
& t_{2}^{2} \omega_{0}^{2}=\tau t_{f}\left(1-k^{2}\right)\left(\omega_{0}^{2}-\gamma_{0}^{2}\right)
\end{aligned}
$$

$t_{f}$ being the $L / R$ time of the control winding and $k^{2}$ is the mutual inductance coupling coefficient between the "shell" and the control winding.

The stability criteria obtained from the Routh array require $D, E, F$, and $D E-C F$ to be positive. This gives the following conditions

$$
\begin{aligned}
& k>\gamma_{0}^{2} / \omega_{0}^{2} \\
& k<\frac{t_{1}}{t_{c}}-\frac{r_{0}{ }^{2} t_{1}{ }^{2} t_{c}}{\omega_{0}{ }^{2} t_{2}{ }^{2} t_{1}}+\frac{t_{1}{ }^{2}}{t_{2}{ }^{2}}
\end{aligned}
$$

and

$$
t_{c}<\frac{t_{j} \omega_{0}^{2}}{\gamma_{0}^{2}}
$$

A direct correspondence between this system and Emmert's is difficult to find however, we do note some similar features that allow us to compare the two.

The gain requirement in Eq. (5.1-23) is similar to the requirement of Eq. (5.1-8) in that the minimum gain must be proportional to $\gamma_{0}{ }^{2}$. The factor relating the two can then be used for the upper gain boundary Eq. (5.1-24) in Emmert!s analysis that accounts for destabilization due to the controller 
causing reversed flow of eddy currents in the "shell."

Recalling $t_{1} w_{0}^{2}$ in Eq. $(5.1-22)$, we see that Eq. $(5.1-25)$ can be reexpressed as

$$
t_{c}<\frac{1}{z_{1}}+\frac{t_{f}\left(k^{2} \omega_{0}^{2}-\gamma_{0}^{2}\right)}{\gamma_{0}^{2}}
$$

This constitutes an improvement over pure time delay where the maximum delay time for $g \rightarrow 1$ became $1 / z_{1}$.

A distinguishing feature between the two systems is the polynomial dispersion relation of Eq. (5.1-20). Since $V$ is always acting for the slightest perturbation $\xi$ (see Eq. (5.1-19)) it is possible to (theoretically) control a change as soon as it occurs. Thus, the plasma is always damped provided conditions of Eq. (5.1-23) thru (5.1-25) are met. Since a practical system requires a finitely detectable disturbance before feedback control can be initiated, it is worthwhile to consider some possible plasma position detectors before presenting a conceptual design.

\subsection{PLASMA POSITION DETECTORS}

The intensity of the Bremsstrahlung radiation varies as $n_{e}{ }^{2} T_{e}^{1 / 2}$ where $n_{e}$ and $T_{e}$ are the electron density and temperature. Because of this weaker de-

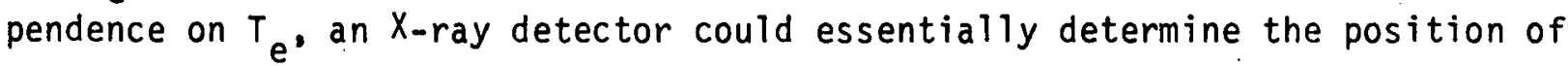
the magnetic axis $R_{m}$ if the currents and density have similar profiles. An $X$-ray detector placed to view constant $z$, however, will see all three peaks if the core of the plasma passes its view. Thus, for vertical control, one could, in principle, determine $\xi \hat{z}$ accurately with a series of $x$-ray detectors. Complications could occur for radial control and the plasma density and current profile would need to be determined beforehand - either by computation or ex-

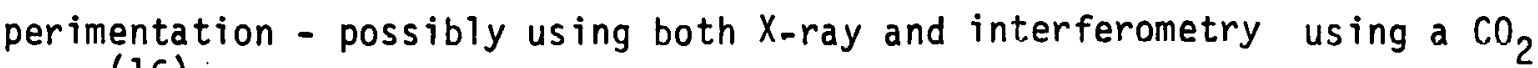
laser (16).

Alcator has used a series of three collimated $X$-ray detectors spaced $4 \mathrm{~cm}$ apart to measure the plasma radial position. By adjusting the vertical field 
after each discharge based on oscillograms from the detector, the burn time was increased from 0.25 to $0.65 \mathrm{sec}$. To improve spatial resolution, more detectors can be used. The sampling time, however, increases to roughly $2 \mathrm{~N} \mu \mathrm{s}$ where $N$ is the number of elements in the array (17). Alcator $C$ is planning to use a series of ten or more detectors with the limiter radius being about $10 \mathrm{~cm}(18)$. Presumably spatial resolution will be $\sim 1 \mathrm{~cm}$ with sampling times $\sim 20 \mu \mathrm{s}$. (These are my estimations and are based on the limiter radius and the sampling time given previously.)

$X$-ray detection is still in its infancy and requires a considerable amount of study and experimentation before becoming applicable to plasma control. American Science and Engineering, Inc. is one of the major companies with research and development in this area. They are presently trying to devise a selfscanning array of $x$-ray detectors with position monitoring and analog and/or digital readout $(16)$.

Magnetic probes placed at different poloidal angles can be used to detect $\dot{B}_{p}$ : the time rate of change of the poloidal magnetic field due to plasma position or current change. ORMAK $(19,20)$ has used the offset signal from probes at $\theta=0$ and $180^{\circ}$ to measure $\Delta$, the shift of the center of the outer flux surface from the center of the conducting shell. After integration this offset signal is:

$$
\frac{u^{-}}{u^{+}}=\frac{B_{p}\left(0^{\circ}\right)-B_{p}\left(180^{\circ}\right)}{B_{p}\left(0^{\circ}\right)+{ }_{p}\left(180^{\circ}\right)}
$$

which can be used to find the shift

$$
\Delta=c_{1}+c_{2} \frac{u^{-}}{u^{+}}+C_{3} \frac{B_{v}+\alpha B_{\text {gap }}}{B_{p}}
$$

where $C_{1}, C_{2}, C_{3}$, and a are experimentally determined constants and $B_{\text {gap }}$ is gap field that leaks through the conducting wall. Using Thompson scattering data to determine the position of the magnetic axis, the magnetic axis offset, relative to the plasma center, was determined and found to agree well with that predicted by theory. The feedback control positioned the plasma radially within a few millimeters during the bulk of the discharge by determining $\Delta$ on-line with analog circuitry and adjusting the vertical field accordingly. 
For vertical motion, a signal similar to Eq. (5.2-1) could be used with probes positioned at $\theta=90^{\circ}$. Toroidal effects would then be absent and the offset signal would be

$$
\frac{u^{-}}{u^{t}}=\frac{\xi}{d}
$$

Where $z= \pm d$ are the positions of the two probes.

The probes used in ORMAK were placed inside the conducting sheli but outside the liner, so their sensitivity was weakened only by the liner and stray fields produced by the vertical field coils. Future tokamaks having a resistive first wall but no liner or conducting shell present problems with either sensitivity (if placed outside the wall) or radiation and thermal damage (if placed inside the wal1). Logical compromises might be to place them in the position of a resistive gap outside the wall or replace them periodically when damaged. Response time appears to be no problem for growth rates larger than the wall time constant if placed outside the wall. If placed at gaps inside the wall, the response would essentially be determined by the amplifier response alone.

Rogowski coils and other magnetic loop detectors can also be used to indicate plasma position and/or plasma current. Cleo-Tokamak (15) used both cosine and stne Rogowski and saddle colls to determine plasma position and current. Ihe cosine Rogowski coil resembles a toroid that wraps around the minor radius of the vessel with turns varying as $\cos \theta$, i.e., weighted at $\theta=0^{\circ}$ and $180^{\circ}$, and is used for horizontal positioning. The sine coil is merely rotated $90^{\circ}$ and is used to determine vertical position. The saddle coil is formed from a rectangular loop that is wrapped around a torus segment as if it were riding it. For horizontal control, the ends of the "saddle" are at $\theta=-90^{\circ}$ with the amplifier connection made at $\theta=+90^{\circ}$. Radial motion of the plasma increases the flux in the segment where $\cos \theta>1$ and decreases the flux where $\cos \theta<1$. For vertical control, the saddle is merely rotated by $90^{\circ}$. Calling $x$ the position variable ( $i . e .$, either $z$ or $r-R_{p}$ ) the integrated response of either saddle or Rogowski coil is of the form

$$
V=A I_{p}\left(x-x^{-}\right)+C B_{z}
$$


where $A, C$, and $x^{-}$are constants determined by calibration and $B_{z}$ is the known vertical field (for vertical motion $C=0$ ). The signals from the two coils are combined to determine $x$ and $I_{p}$. The position and plasma current then determine the stabilizing field required. The detection time is set by the wall diffusion time $(\sim 1 \mu \mathrm{s})$ in the Cleo-Tokamak. A transistor amplifier network changes the current in four control loops that produces the stabilizing field.

Radial control for a vertically stable plasma increased the discharge time from $0.11 \mathrm{sec}$ to $0.2 \mathrm{sec}$ and the plasma remained centered to $\pm 1 \mathrm{~cm}$ for $0.14 \mathrm{sec}$. A vertically unstable plasma was also stabilized and centered for up to $0.1 \mathrm{sec}$. The experimentally determined gain boundaries for this vertical control, however, were about a factor of four below those given in Eqs. (5.1-23) and (5.1-24).

$X$-ray detectors, magnetic probes, Rogowski coils, and saddle coils are the major plasma position (and current) detectors considered for feedback systems today. The latter three are state-of-the-art devices but subject to sensitivity problems and stray magnetic fields. The $x$-ray detector is relatively new and requires more complicated circuitry and still much development. It is the only device, however, that is capable of determining the plasma position, in principle, absolutely. For this reason and for its fast response, it is likely to be the candidate detector for future tokamaks. However, the other devices, despite their drawbacks, have proven themselves in the systems mentioned as well as others and should not be ruled out altogether for future tokamaks.

\subsection{DESIGN FOR FEEDBACK CONTROL OF VERTICAL INSTABILITY IN TNS}

In this final section, we present the necessary features of the control coils to feedback stabilize the vertical instability. Amplifier and detector specifications are not given, but the power requirements for the overall system with and without feedback delay are calculated. We assume that the detectors are capable of detecting a vertical shift $\sim 5 \mathrm{~cm}$ so the maximum plasma excursion is controlled to $\pm 0.1 \mathrm{~m}$.

The control coils are located near or in the same casings as the EF coils D-5, $0-6$, and $0-7$. Control by varying EF coils currents will require much larger 
power supplies than these separate coils would. This will be demonstrated in the analysis.

The EF coil Configuration II will be used for design purposes since this contracted 1-D-0 grouping is the more practical and economical configuration.

The design will work also for Configuration $I$, however, since it is more stable than II.

Using a decay index of -0.8 and $B_{z}=0.6 \mathrm{~T}$, the minimum gain is (by Eq. (5.1-8))

$$
G_{\min }=0.096 \mathrm{~T} / \mathrm{m}
$$

Since we wish to stabilize the transient response when delay time is included, we choose

$$
G=0.15 \mathrm{~T} / \mathrm{m}
$$

or

$$
g=\frac{G}{G_{\text {min }}}=1.56
$$

For effective coil parameters $\omega_{0}^{2}=2.64 \times 10^{11} \mathrm{sec}^{-2}, \tau=2.5 \mathrm{sec}$, and $\gamma_{0}{ }^{2}=$ $1.35 \times 10^{11} \mathrm{sec}^{-2}$, the damped rates for "instantaneous" feedback are (by Eqs. $(5.1-9)$ and $(5.1-10))$

$$
\begin{gathered}
s_{1}=-0.27 \mathrm{sec}^{-1} \\
s_{2,3}= \pm i\left(3.6 \times 10^{5}\right)-0.065 \mathrm{sec}^{-1}
\end{gathered}
$$

The growth rate without feedback is $z_{1}=0.418 \mathrm{sec}^{-1}$ (or rising time $=2.4 \mathrm{sec}$ ) so as an indication of "instantaneous" we choose a feedback system rise time of $t_{r}=\frac{1}{10 z_{1}}=0.25 \mathrm{sec}$. Thus, the plasma will deviate no more than $e^{0.1}=1.1$ times the initial detected plasma deviation $\xi_{0}$. Choosing $\xi_{0}=0.1 \mathrm{~m}$ for instantaneous feedback, the maximum radial stabilizing field will be

$$
B_{r \max }^{f . b .}=G \xi_{0}=0.015 \mathrm{~T}
$$

inwardly directed for the positive perturbation with ${ }_{p}$ in the negative sense. 
Since $\sin \theta_{j}>0.9$ for coils 5,6 , and 7 , let us choose, for simplicity, a maximum field contribution $B_{r_{j}}=0.005 \mathrm{~T}$ from each of the three coil pairs.

Denoting $n_{i}$ as the number of turns in either top or bottom control coil and $i_{i}$ as the maximum current in the control coil, the $n_{i} i_{i}$ (amp turns) required can be balanced according to

$$
\frac{A_{i}}{\mu} \frac{n_{i}{ }^{i}}{N_{i}}=\frac{I p}{m} B r_{i}
$$

where $A_{i}$ and $N_{i}$ are the values given in Table 4.2-2. This balance gives the following amp-turns in the coils:

$$
\begin{aligned}
& n_{5} i_{5}=54.2 \mathrm{kA} \\
& n_{6} i_{6}=39.6 \mathrm{kA} \\
& n_{7} i_{7}=32.0 \mathrm{kA}
\end{aligned}
$$

which also appear in Figure 2.2-1.

To determine the controller voltage, we need to know the single turn inductances $m_{i j}=m_{i j}-m_{i-j}$ for the control coils. The single turn mutual inductances can be found by dividing the elements in Table 4.2-1 by $N_{i} N_{j}$. The single turn self-inductances $i=j$, however, are less well defined since the self-inductance depends on the coil cross section dimensions. But this dependence is only logarithmic so to a reasonable approximation $m_{i j}=M_{i j} / N_{i} N_{j}$ for all cases.

We shall assume that the currents in the coils rise linearly in a time $t_{r}$ and that induced currents in the EF coils can be neglected. The latter approximation is a poor one when the currents $i_{j}$ are beginning their ramp. As they approach their maximum value, however, the induced EF coil currents will stop and reverse direction. Their effect, in a sense, averages out and we may approximate for the maximum controller voltage at 5 (see circuit schematic in Figure 5.3-1).

$$
v_{5}=n_{5} i_{5} \frac{2 R_{5}}{N_{5}}+\frac{2 n_{5}^{2} m_{55} i_{5}}{t_{r}}+\frac{2 n_{5} n_{6} m_{56}^{i_{6}}}{t_{r}}+\frac{2 n_{5} n_{7} m_{57} i_{7}}{t_{r}}
$$




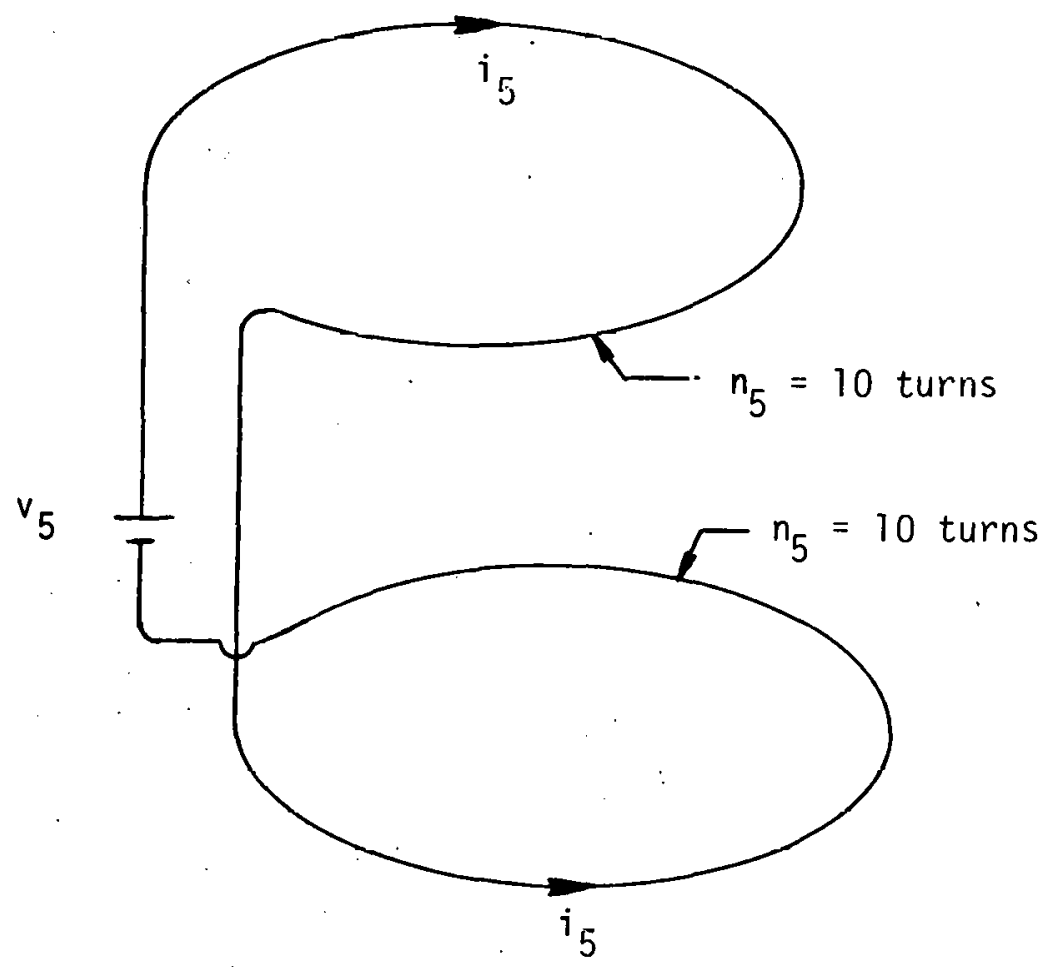

Figure 5.3-1. Typical Control Circuit for Feedback Control System Illustrating Series Connection for Controller. 
assuming the only resistance in the circuit is the resistance of the coil itself. The circuit equations for 6 and 7 are similar to the one for 5 .

Denoting the maximum power in the circuit 5 as $P_{5}=v_{5}{ }_{5}$ (etc for 6 and 7 ) the following expressions result for the three controller groups

$$
\begin{aligned}
& P_{5}=\frac{0.150 \mathrm{MJ}}{t_{r}}+\frac{2.39 \mathrm{MW}}{n_{5}} \\
& P_{6}=\frac{0.128 \mathrm{MJ}}{t_{r}}+\frac{1.56 \mathrm{MW}}{n_{6}} \\
& P_{7}=\frac{0.094 \mathrm{MJ}}{t_{r}}+\frac{1.19 \mathrm{MW}}{\mathrm{n}_{7}}
\end{aligned}
$$

Thus, we see that the magnetic power is independent of coil turns but the ohmic power is inversly proportional.

To keep the size of the control coils as small as possible without adding appreciable joule heat, let us take $n_{5}=n_{6}=n_{7}=10$. Taking $t_{r}=0.25 \mathrm{sec}$, Eq. $(5.3-10)$ gives

$$
\begin{aligned}
& P_{\text {mag }}=1.49 \mathrm{MW} \\
& P_{J H}=0.51 \mathrm{MW}
\end{aligned}
$$

as the magnetic and joule-heat powers, respectively. The total controller power is then

$$
P=P_{\text {mag }}+P_{J H}=2.0 \mathrm{MW}
$$

for the "instantaneous" feedback.

To offer a more optimum system with feedback delay we shall design with Eqs. $(5.1-15)$ thru $(5.1-18)$ in mind. 
The time delay is given by Eq. $(5.1-16)$

$$
t_{d}=\frac{0.426}{0.418}=1.02 \mathrm{sec}
$$

and $g=1.56$ satisfies Eq. (5.1-17). For long term stability, Eq. (5.1-14) must be satisfied. This gives $t_{d}<1.75 \mathrm{sec}$ which is acceptable.

We would like to design to $\xi_{\max }=0.1 \mathrm{~m}$ so $\xi_{0}=0.1 / 1.54=0.065 \mathrm{~m}$ makes a constraint on the detectors that hopefully can be met.

The parameter $U$ in Eq. $(5.1-18)$ can be found using

$$
E_{\text {mag }}=1 / 2 \sum_{i, j=-5}^{7} m_{i j} n_{i} n_{j} i_{i} j_{j}
$$

which is already available from the power value $P_{\text {mag }}$ by

$$
E_{\text {mag }}=\frac{P_{\text {mag }} t_{r}}{2}=0.186 \mathrm{MJ}
$$

Thus,

$$
U=\frac{E_{\text {mag }}}{B_{r_{\text {mag }}}^{f . b .}}=826 \frac{M J}{T^{2}}
$$

giving a magnetic power optimum by Eq. (5.1-18)

$$
P_{\text {mag }}=0.154 \mathrm{MW}
$$

The total power with joule heat included is then

$$
P=P_{\text {mag }}+P_{J H}=0.67 \mathrm{MW}
$$

about a third of the previous value.

It is important to note that the analysis offered in this section is based on many approximations. Where possible, however, the worst case was assumed, so the power requirements should be no greater than indicated. 
If the EF currents were adjusted to provide feedback, the controller voltages given by Eq. (5.3-9) would be roughly the same, but the controller power supply would have to carry the equilibrium current $(-0.82$ MA-turns for D-5) in addition. The magnetic power $P_{5}$ would then be a ratio about $\frac{820}{54}=15$ times as great. The ohmic increase would be $15 \frac{n_{5}}{N_{5}} \simeq 3.4$ times as great. These increases result in controller power specifications of roughly, 25 MW for "instantaneous" control and $4 \mathrm{MW}$ with delayed control. Thus, the required power would increase by roughly a factor of ten without separate controllers.

Since $I_{p}=6.1 \mathrm{MA}$ can be regarded as the upper limit in TNS, it is beneficial to know the power required by the controller at other currents. This power varies as $\left(B_{r}^{f . b} \cdot\right)^{2}$ which varies as $B_{z}^{2} \sim I_{p}^{2}$. So the $5 \%$ B at 4.3 MA will draw half as much peak power as at 6.1 MA provided the decay index remains relatively constant.

Induced currents due to the coupling between the $\mathrm{OH}$ controller and EF controller during the start of the discharge might be expected. However, the top controller loops are positively coupled to the top $\mathrm{OH}$ and EF coils and the bottom loops are negatively coupled to the bottom $\mathrm{OH}$ and $E F$ coils so the effect of induced currents cancels. The exception is the EF-D coils which are coupled in the opposite sense. Induced currents here, of course, also cancel. Thus, unwanted induced currents due to EF controller or the $\mathrm{OH}$ controller coupling can be neglected irregardless of whether the $E F$ and $O H$ are hooked in a series or parallel connection.

Induced currents due to plasma motion and EF controller coupling is expected during a vertical perturbation if the EF coils are placed in parallel. This effect, however, is desired since the induced EF coil currents were found to reduce the vertical instability significantly.

It is desirable to compare the gain and delay time requirements found by Emmert's method to those of Hugill and Gibson. The condition in Eq. (5.1-23) 
gives a minimum gain of 0.51 which is a factor of two lower than the value of the minimum relative gain $g>1$.

To compare these two methods, the coupling coefficient for mutual inductances, $k$, must be found. For two concentric shells, this coupling coefficient varies as the ratio of their wall. radii. Since, however, $\omega_{0}{ }^{2}$ varies inversly as $\rho_{w}{ }^{2}$, an estimate of $k^{2}$ can be obtained by finding the ratios of $\omega_{0}^{2}$. Since the effective $\tau$ and $\omega_{0}{ }^{2}$ were easy to define for the EF coil Configuration III and did not differ more than $10 \%$ with those values for I and II, effective $\omega_{0}^{2}$ and $\tau$ for the control coils can be estimated in a similar manner using Eqs. (4.1-31) thru $(4.1-33)$. These give $k^{2}, w_{0}^{2}$, and $t_{f}$ in $E q .(5.1-22)$ as

$$
\begin{aligned}
k^{2} \omega_{0}^{2} & =1.8 \times 10^{11} \mathrm{sec}^{-2} \\
t_{f} & =0.74 \mathrm{sec}
\end{aligned}
$$

The values of $t_{1}$ and $t_{2}$ from Eq. (5.1-22) are then $t_{1}=1.35 \mathrm{sec}$ and $t_{2}=0.54$ $\mathrm{sec}$. These require a controlier time constant $t_{c}<2: 6 \mathrm{sec}$ by Eq. $(5.1-25)$.

As $t_{c} \rightarrow t_{c \text { max }}$, the 1 imit on the gain $K_{\max }$ in $E q .(5.1-24)$ approaches $K_{\min }$. As $t_{c} \rightarrow 0$, however, $K_{\text {max }} \rightarrow \infty$ and there is no limit on the gain. To compare the two methods, let us take $t_{c}=t_{d}=1 \mathrm{sec}$. Then, the following limit is imposed upon $\mathrm{K}$ :

$$
0.51<K<5.3
$$

and if g scales proportionally

$$
1<g<10
$$

This seems to indicate that our system is stable against the possibility of reversed eddy currents driving the plasma unstable in the opposite direction.

The roots to $E q .(5.1-20)$ for $t_{c}=1 \mathrm{sec}$ and $k=0.8$ (i.e., $1.56 \mathrm{~K}_{\text {min }}$ ) are 


$$
\begin{aligned}
& z_{1}=-5.1 \mathrm{sec}^{-1} \\
& z_{2,3}= \pm i(0.37)-0.26 \mathrm{sec}^{-1}
\end{aligned}
$$

Note that the damping rates are greater than or of the same order of magnitude as those predicted by Eq. (5.3-4) and (5.3-5) for "instantaneous" feedback. The high frequency oscillations in Eq. (5.3-5) are absent here since the assumption $\left|z^{2}\right|<\gamma_{0}^{2}$ was used to reduce Eq. (5.1-20) to its third order (instead of fifth order) form. 


\subsection{CONCLUSIONS}

To summarize the control of the vertical instability in TNS, we have seen how a plasma with a rising time of $3 \mu \mathrm{s}$ (without eddy currents considered) could be slowed down to $\sim 30 \mathrm{~ms}$ by the first wall provided it is continuous in the toroidal direction. Different parallel EF coil configurations were found to slow the instability down to rising times $\sim 2$ to $3 \mathrm{sec}$.

The "shel1-1ike" behavior of the discrete EF coils allowed considerable simplification of the control theory needed to design a feedback system to fully stabilize the vertical instability.

The power requirements for a system using three separate control circuits to drive the feedback currents are very modest - about 2 MW for "instantaneous" control and less than $1 \mathrm{MW}$. for a $1 \mathrm{sec}$ delay.time. 


\subsection{REFERENCES}

1. Y-K M. Peng and D. J. Strickler, "Poloidal Field System Design for TNS," ANS :Transactions 27 (1977) 29.

2. H. P. Furth, Tokamak Research (Review Paper), Nucl. Fusion 15 (1975) 487.

3. J. A. Wesson, Hydromagnetic Stability of Tokamaks (Review Paper), Nucl. Fusion 18 (1978) 87 .

4.. 0. Gruber and R. Wilhelm, The Belt Pinch - A High B Tokamak With NonCircular Cross Section, Nucl. Fusion 16 (1976) 243.

5. G. Laval, R. Pellat, and J. S. Soule, Hydromagnetic Stability of a CurrentCarrying Pinch With Non-Circular Cross Section, Phys. Fluids 17 (1974) 835.

6. E. Rebhan, Stability Boundaries of Tokamaks With Respect to Rigid Displacements, Nucl. Fusion 15 (1975) 277.

7. V. S. Mukhovatov and V. D. Shafranov, Plasma Equilibrium in a Tokamak (Review Paper) Nucl. Fusion 11 (1971) 605.

8. F. A. Haas, Stability of a High \& Tokamak to Uniform Vertical Displacements, Nucl. Fusion 15 (1975) 407.

9. W. R. Smythe, Static and Dynamic Electricity, 3rd Edition, McGraw-Hill (1968) 290-291,

10. J. D. Jackson, Classical Electrodynamics, 2nd Edition, John Wiley \& Sons, Inc. (1975) 177-180.

11. J. J. DiStefano, A. R. Stubberud, and I. J. Williams, Feedback and Control Systems, Schaum's Outline Series, McGraw-Hill (1967). 87 .

12. Y-A. Chao, E. R. Frantz, and T-F Yang, "A Practical Method of Analyzing the the Effects of Induced Eddy Currents on an Unstable Plasma Displacement of a Rigid Plasma," to be published in the Proc. of the 3rd Topical Meeting on Tech. of Conf. Nucl. Fusion, Santa Fe (May 1978).

13. J. R. LaMarsh, Introduction to Nuclear Reactor Theory, Addison Wesley (1966) 422-425.

14. G. L. Kulcinski, et al., "TETR-A Tokamak Engineering Test Reactor for Quality Materials and Blanket Components for Early DT Fusion Power Reactors," Proceedings of the Second Topical Meeting on the Technology of Controlled Nuclear Fusion, Vol I, p 73 (Richland, Washington) 1976. 
15. J. Hugill and A. Gibson, Servo-Control of Plasma Position in Cleo-Tokamak, Nucl. Fusion 14 (1974) 611 .

16. T. N. Edelbaum, P. A. Madden, R. E. Vov, R-1046-Rev 1, Fusion Reactor Control Study, First Annual Report, EPRI Program RP546-2 (July 1977).

17. P. A. Madden, Investigation of Plasma Position Control and Sensors Requirements for Tokamak EPR's (1977).

18. W. W. Saylor, Plasma Position Control in a Tokamak Experimental Power Reactor, MIT Master's Thes is (January 1977).

19. J. L. Anderson, et al., Feedback Control for Plasma Equilibrium in ORMAK, Nucl. Fusion 16 (1976) 629.

20. R. V. Miskell, A Control Strategy for Plasma Equilibrium in a Tokamak, Report Y-1988 Oak Ridge Y-12 (August 1975). 